\title{
Distribution of the high-velocity clouds in the Galactic halo
}

\author{
C. A. Olano` \\ Universidad Nacional de La Plata, Facultad de Ciencias Astronómicas y Geofísicas, Paseo del Bosque, 1900 La Plata, Argentina \\ e-mail: colano@lilen.fcaglp.unlp.edu.ar
}

Received 27 March 2007 / Accepted 19 March 2008

\begin{abstract}
Context. There is a connection between the kinematics and sky distribution of the high velocity clouds (HVCs) and the spatial velocity and orbital plane of the Magellanic Clouds (MCs) that allow us to conclude that most HVCs were ejected from the Clouds, some hundreds of Myrs ago, probably by an encounter between the Large (LMC) and Small Magellanic Cloud (SMC).

Aims. Based on the idea that HVCs have progenitors in the MCs, we attempt determine theoretical distances to the HVCs using observed data of the sky positions and radial velocities, and thus to map the spatial distribution of the HVCs.

Methods. i) In the context of a kinematical model we have found an analytical formula that gives the distance of an HVC; ii) we have developed two dynamical models in order to study the orbits of the HVCs under the gravitational forces exerted by the Galaxy and the MCs and forces of resistance due to the movement of the HVCs through the Galactic gaseous disk. We have adopted the gravitational potential of the Milky Way given by a dark matter halo (Model 1) plus a massive thick disk partially composed by dark matter (Model 2). By means of a fitting to certain Galactic, MC and HVC conditions we have obtained values for the free parameters of the Models. We have obtained the dynamical distances to the HVCs by constructing the theoretical relationship between the radial velocity and the distance in the line of sight of each HVC.

Results. The HVCs can be divided into three major groups: Population MS, Population W and Population A-C. Population MS comprises the Magellanic stream and the leading arm of the Magellanic system. The HVCs of this population are dispersed around the MCs within a volume of $\approx 200 \mathrm{kpc} \times 200 \mathrm{kpc} \times 60 \mathrm{kpc}$. Population W is situated at a mean distance of $\approx 15 \mathrm{kpc}$ from the Sun, and has been braked by the gas of the Galactic disk. Population A-C includes Complexes A, C, H, L, M, and ACHVC. The dynamical models give two solutions for the distance of each HVC of Population A-C. The near-distance solutions place Population A-C at $\approx 6 \mathrm{kpc}$ from the Sun, showing that this chain of HVCs started to interact with the outer parts of the Galactic gaseous disk about 100 Myr ago. While, the spatial distribution of Population A-C represented with the far distances has the shape of a gigantic ring with a radius of $\approx 125 \mathrm{kpc}$. Our results support the idea that the LMC-SMC encounter triggered a period of star formation bursts, in which part of the LMC bar was probably formed. The interactions of a few $10^{5}$ massive stars formed in this era with the then-existing low metallicity ISM of the MCs would have created the HVCs
\end{abstract}

Key words. ISM: clouds - galaxies: Magellanic Clouds - Galaxy: halo - galaxies: interactions

\section{Introduction}

The high-velocity clouds (HVCs) are diffuse emission structures, which were first observed through the $21 \mathrm{~cm}$ line of HI, that have velocities forbidden by Galactic rotation. They are located outside the Galactic plane and are well-distributed in the southern and northern hemispheres. The origin and nature of the HVCs have intrigued astronomers since the discovery of the clouds in 1963. Since then, numerous explanations for their origins have been proposed. The principal obstacle to a convincing interpretation is the lack of knowledge of their distances. For a summary of recent progress in understanding HVCs, we refer to the review by Wakker \& van Woerden (1997) and volume 312 of the Astrophysics and Space Science Library, which was dedicated to the HVC phenomenon (van Woerden et al. 2004).

The idea of a Galactic origin for the majority of the HVCs, as proposed by the "Galactic fountain" model (Shapiro \& Field 1976; Bregman 1980), appears to be incompatible with the low metallicity nature of the HVCs, and the fact that the HVCs do not follow the Galactic rotation. The hypothesis that HVCs are

\footnotetext{
* Member of the Carrera del Investigador Científico del CONICET, Argentina.
}

members of Local Group galaxies was rejected by Oort (1966), on stability arguments, and reconsidered by Blitz et al. (1999) within the framework of a cosmological theory for hierarchical galaxy formation, in which the dynamical mass is dominated by cold dark matter. The non-detection of HVCs in galaxy groups analogous to the Local Group (Zwaan 2001; Pisano et al. 2004) and the fact that there are no stars in HVCs (Siegel et al. 2005), including compact HVCs (Simon \& Blitz 2002; Hopp et al. 2007), throw doubt on the extragalactic interpretation of the HVCs.

In contrast evidence in favor of a circumgalactic location for the HVCs, continues to be discovered (Peek et al. 2006; Thom et al. 2007). Oort defended the circumgalactic hypothesis by proposing that the HVCs are clouds of Galactic gas lying inside the halo. To explain the anomalous velocities of HVCs, he speculated that gas in the Galactic halo is projected inwards by intergalactic winds (Hulsbosch \& Oort 1973). Maller \& Bullock (2004), Sommer-Larsen (2006), and Peek et al. (2007) developed new models, in which the Galaxy is surrounded by a halo of hot gas, where the thermal instabilities lead to the formation of warm clouds that rain onto the Galactic disk as HVCs. Our knowledge of the physical conditions in the Galactic halo is insufficient to decide whether the requirements of these models 
are fulfilled. Within the framework of these models, it is difficult to explain why the majority of the HVCs have the similar kinematics to the Magellanic Stream (MS). The most simple explanation is that HVCs are part of stream of clouds, in which the Milky Way is immersed (Olano 2004, hereafter Paper I). Hence, the problem of the origin and nature of the HVCs might be reduced to the problem of finding the processes that generated the MS. The possibility that most HVCs are associated with the MS was first suggested by Giovanelli (1981) and Mirabel (1981).

It is almost certain that the MS consists of gas removed from the Magallanic Clouds (MCs). Previous studies have focused on two mechanisms that could have extracted gas from the MCs: ram pressure force which acts as the MCs move through the hot gaseous halo of the Galaxy (Meurer et al. 1985; Moore \& Davis 1994), and tidal forces between the Small Magellanic Cloud (SMC), the Large Magellanic Cloud (LMC), and the Galaxy (Gardiner \& Noguchi 1996; Yoshizawa \& Noguchi 2003; Connors et al. 2006; Bekki \& Chiva 2007). The combined effects of both mechanisms were studied by Mastropietro et al. (2005). These models fail to reproduce, however, several characteristics of the stream; for a critical discussion, see Nidever et al. (2007). The facts can be better explained if we consider that the primary mechanism is one of gas expulsion from the MCs, due to internal processes in the MCs. With a simple simulation of the ejection process of the HVCs, and of the subsequent dynamical evolution of the HVC system through the Galactic halo, we showed in Paper I that this model can reproduce the main structural and kinematical features of the MS and the remainder of the HVCs. The gas expulsion that created the HVCs was probably caused by winds and explosions of massive stars formed in a major starburst, which was triggered by the LMC-SMC encounter (Paper I).

We determine theoretical distances to HVCs, by developing two methods based on a model for the kinematics and dynamical evolution of the system of HVCs presented in Paper I. This model is improved by using a gravitational potential for the Galaxy's halo whose main parameters are estimated using the observed data of the MCs and HVCs. Applying these methods, we obtain the distances to the HVCs of Wakker's catalog; see Wakker (2004) for more information on the catalog properties and references to its original sources.

In Sect. 2, we outline the evolution of the system of HVCs by means of a simple kinematical model. This model assumes that the velocity of an HVC is composed of a systemic velocity of translation, and a falling velocity due to the Galactic gravitation, both of which are common to all HVCs, and in addition a proper velocity of expansion due to the initial kinetic energy of the HVC. In this model, the HVC system evolved from a relatively small volume centered on the LMC, reaching the present size in an interval of time $T_{\mathrm{k}} \approx 550 \mathrm{Myr}$. In Sect. 3, we present the dynamical models and the adjustment of their free parameters: Sects. 3.1 and 3.1.1 concern Model 1, Sects. 3.2 and 3.2.1 Model 2, and Sects. 3.3 and 3.3.1 the corresponding method to obtain the theoretical distances to the HVCs and its application to Complexes A and C. In Sect. 4, we give the results of Model 1, without (Sect. 4.1) and with (Sect. 4.2) drag forces. Then, in Sect. 5 we discuss the low-metallicity nature of the HVCs in the framework of our models and the possible processes that created HVCs. Finally, in Sect. 5 we give a summary and conclusions of this work.

\section{Kinematical model}

We will try to present here a simplified analysis of the kinematics of the system of HVCs which might help us to solve the problem of obtaining the distance to an HVC from knowing solely its sky position and radial velocity.

According to Paper I, the Galaxy is surrounded by a large cloud of HVCs centered on the MCs, i.e. on the Galactic position $\left(l_{\mathrm{c}}, b_{\mathrm{c}}\right)=\left(280^{\circ} 5,-33^{\circ} .9\right)$ at a distance from the Sun $d_{\mathrm{c}}=50.1 \mathrm{kpc}$. This metacloud is translating as a whole with a barycenter velocity similar to the spatial velocity of the MCs, since the HVCs were launched from this moving platform, and expanding from this center due to the original velocities with which the HVCs were ejected from the Clouds. By means of a simplified model of the kinematics of the system of HVCs, we will derive an analytic formula relating the radial velocity of an HVC to its distance.

Denoting the translation velocity of the cloud of HVCs with respect to the Galactic center by $V_{\mathrm{f}}$, the radial velocity of an HVC due to this translation movement of the system is

$\rho_{\text {tran }}=-V_{\mathrm{f}} \cos \left(l-l_{0}\right) \cos \left(b-b_{0}\right)$,

where $(l, b)$ and $\left(l_{0}, b_{0}\right)$ are respectively the Galactic coordinates of the $\mathrm{HVC}$ and of the radiant point, i.e. the direction from which the stream of HVCs comes.

Let us consider a simple model of free expansion for the system of HVCs, ignoring for the moment the action of the Galactic gravitational potential and braking forces of the gaseous medium in the halo. Then the present velocity of expansion $V_{\exp }$ of an $\mathrm{HVC}$, with respect to the center $\mathrm{C}$ of the system of HVCs, is considered equal to its initial velocity with which was ejected from the MCs. Hence

$\boldsymbol{V}_{\exp }=k \boldsymbol{r}_{\mathrm{c}}$

where $\boldsymbol{r}_{\mathrm{c}}$ is the position vector of the HVC with respect to the expansion center $\mathrm{C}$ of the system of HVCs and $k$ is a constant related to the time $T$ elapsed since the moment of the ejections by $1 / T$. In other words, the expansion velocity of an HVC is proportional to the distance $\left|\boldsymbol{r}_{\mathrm{c}}\right|=r_{\mathrm{c}}$ between the HVC and C, i.e. the MCs. Since we are interested in knowing the distances to the HVCs from the Sun, we will express $r_{\mathrm{c}}$ in Eq. (2) as a function of the HVC's distance $d$ from the Sun through the trigonometric expression $r_{\mathrm{c}}^{2}=d^{2}+d_{\mathrm{c}}^{2}-2 d d_{\mathrm{c}} \cos \alpha$, where $\alpha$ is the angle between the direction to $\mathrm{C}$ and the direction to the HVC. The scalar product $\boldsymbol{d} \cdot \boldsymbol{d}_{\mathrm{c}}$ gives us $\cos \alpha=\cos b \cos b_{\mathrm{c}} \cos \left(l-l_{\mathrm{c}}\right)+\sin b \sin b_{\mathrm{c}}$. The radial velocity component due to the expansion is obtained by projecting $\boldsymbol{V}_{\exp }$ on the line of sight, $\rho_{\exp }=\left|\boldsymbol{V}_{\exp }\right| \cos \beta$, where $\cos \beta=\sqrt{1-\left(\frac{d_{\mathrm{c}}}{r_{\mathrm{c}}}\right)^{2} \sin ^{2} \alpha}$. Making all the replacements we obtain,

$\rho_{\exp }=k d-k d_{\mathrm{c}}\left(\cos b \cos b_{\mathrm{c}} \cos \left(l-l_{\mathrm{c}}\right)+\sin b \sin b_{\mathrm{c}}\right)$.

Thus the theoretical LSR radial velocity of an HVC is given by

$\rho=-V_{\mathrm{s}} \sin l \cos b+\rho_{\text {tran }}+\rho_{\text {exp }}-V_{\text {fall }}$,

where the first term corresponds to the component of the solar motion $\left(V_{\mathrm{s}}=220 \mathrm{~km} \mathrm{~s}^{-1}\right)$ in the direction $(l, b)$ of the HVC, and $V_{\text {fall }}$ is a correction for the dynamical effects and can be interpreted as a velocity component of the HVC added in the direction from the HVC to the Galactic center by the action of the gravitational force of the Milky Way. Thus, $V_{\text {fall }}$ tends to point in the direction of the Galactic center and hence $\boldsymbol{V}_{\text {fall }}$ projected on the line of sight $\approx\left|\boldsymbol{V}_{\text {fall }}\right|=V_{\text {fall }}$; a good approximation if $d \gg r_{\mathrm{s}}$, where $r_{\mathrm{s}}=8.5 \mathrm{Kpc}$ is the distance between the Sun and the Galactic center. 

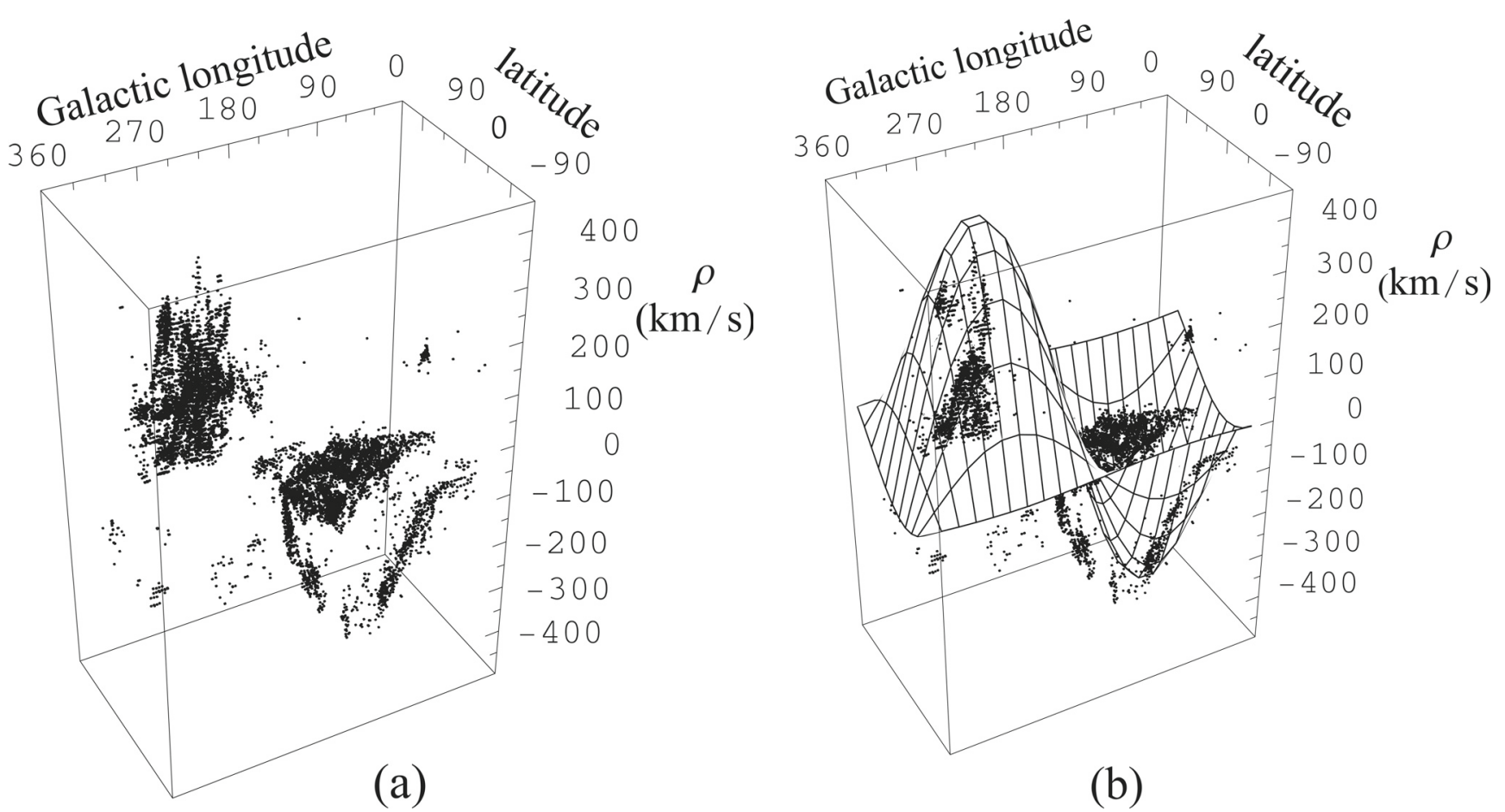

(b)

Fig. 1. a) Distribution of the observed radial velocities of the HVC components a function of the Galactic latitudes and longitudes. b) Same as a) plus a surface that represents the theoretical radial velocities of the HVCs at a given distance $(d=70 \mathrm{kpc})$.

By comparing the $\rho-d$ relationships of Eq. (4) for different positions $(l, b)$ with those corresponding to the dynamical models (next sections), we obtained the best fit parameters for Eq. (4): $k=(1 / 550) \mathrm{km} \mathrm{s}^{-1} \mathrm{pc}^{-1}, V_{\mathrm{f}}=282 \mathrm{~km} \mathrm{~s}^{-1}$, $V_{\text {fall }}=133 \mathrm{~km} \mathrm{~s}^{-1}, l_{0}=78^{\circ}$ and $b_{0}=2^{\circ}$. With the observed radial velocity and Galactic coordinates of an HVC, Eq. (4) can be solved for $d$, the kinematic distance to the HVC (i.e. $d=f(l, b, \rho)$ ). Using Eq. (4), we will obtain the kinematic distances to the HVC components of Wakker's catalog. This cata$\log$ gives, for each HVC component detected in the HI spectra, the longitude $l$, the latitude $b$ and the LSR radial velocity $\rho$, etc. These observational data are shown in Fig. 1 where each point represents the elemental set $(l, b, \rho)$ of a HVC component. The theoretical $\rho$ depends on distance $d$ as well as on $l$ and $b$. To compare with the observations, Fig. 1 shows the theoretical $\rho$ as a function of $l$ and $b$ for $d=70 \mathrm{kpc}$. Figure 2 displays the spatial distribution of the HVC components derived from Eq. (4) with the observational data plotted in Fig. 1. The formula transforming the $(d, l, b)$ spherical coordinates centered on the Sun into the $(X, Y, Z)$ Cartesian coordinates centered on the Galactic center is given in Sect. 3.3 (Eq. (18)). Most of the points in the $(l, b, \rho)$ space of Fig. 1 can be converted into points of the $(X, Y, Z)$ physical space (Fig. 2).

\section{Dynamical models}

In order to derive the spatial distribution of the HVCs, we first need to obtain the origin time and position of the HVCs. They are common to all HVCs, and together with the radial velocity and sky position of each HVC will give us the necessary boundary conditions to calculate the orbit of each HVC. In our model, the origin time and position of the HVCs are coincident with the time and position of the collision between the LMC and the SMC. There are physical evidences that this collision really happened and triggered the processes that gave origin to the HVCs. Hence, to obtain the initial conditions of the HVCs, we

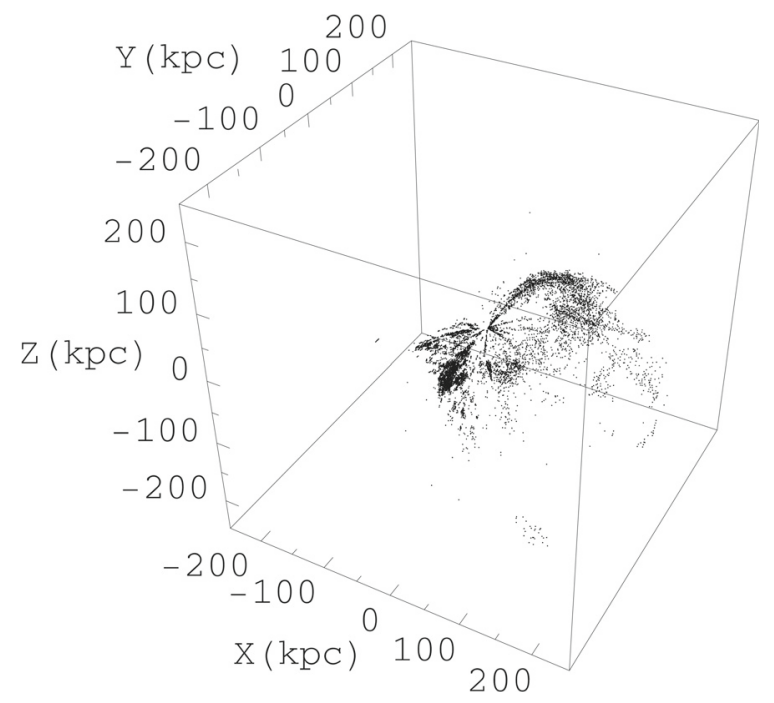

Fig. 2. Spatial distribution of the HVC components derived from the analytical formula (4) with the Galactic co-ordinates and radial velocities of the HVC components cataloged by Wakkker. This Cartesian coordinate system $(X, Y, Z)$ has the origen at the Galactic center, the $X$-axis pointing in the direction of the Sun's Galactic rotation, the $Y$-axis pointing in the direction from the Galactic center to the Sun, and the $Z$-axis pointing toward the Galactic north pole

will trace back in time the orbit of the LMC and of the SMC governed by the Milky Way's gravitational potential. With this purpose we need the present positions and spatial velocities of both Clouds and a law for the force field of the Galactic potential. However, some observational data such as the proper motion of the SMC, from which the SMC's spatial velocity is derived, and the Galactic potential are not accurately determined. Even so, these parameters of uncertain values can be adjusted by requiring the fulfillment of two conditions: (Condition 1) the proper 
motion components of the SMC and the prescribed force law for the Galactic potential should make possible the encounter of the Clouds in the past; (Condition 2) the distance predicted by the model for an HVC with known distance (a component of Complex A) should agree with its distance determined by direct methods.

Recent studies by de Boer et al. (1997), Kalberla (2003) and Kalberla et al. (2007) have show that the Galactic potential is more complex than considered before. A detailed treatment of the Galactic potential is beyond the scope of this work. For our present aim, however, it is enough to describe this potential with relatively simple models. We will consider two models, Model 1 in which the Galactic potential is due to a dark matter halo, and Model 2 in which the additional potential of a massive disk is taken into account. We will demonstrate that the results of Model 2 are coherent only if the visible disk of the Milky Way is associated with a massive and relatively thick disk of dark matter, in agreement with the study of Kalberla et al. (2007).

\subsection{Model 1}

The Galactic dynamics is essentially governed by the mass distribution of the dark matter component. In first approximation, the movements of the HVCs and of the MCs can be formally described in terms of the Galactic gravitational potential as due to a massive spherical halo of dark matter. Then, the global force field of the Galaxy can be represented in relatively simple terms of a central field attributed to the dark halo. Hence the motion equations of a particle representing an $\mathrm{HVC}$, or portion of this (i.e. an HVC component), under this central gravitational field are given by

$\ddot{\boldsymbol{r}}+\frac{1}{r} \frac{\partial \phi_{\mathrm{h}}(r)}{\partial r} \boldsymbol{r}=0$

where $\phi_{\mathrm{h}}$ is the potential of the dark halo and $\boldsymbol{r}$ the position vector whose components are expressed in a Cartesian coordinate system $(X, Y, Z)$ with the origin at the Galactic center, the $X$-axis pointing in the direction of the Sun's Galactic rotation, the $Y$-axis pointing in the direction from the Galactic center to the Sun, and the $Z$-axis pointing toward the Galactic north pole.This Galactocentric rest frame of reference will be used throughout the paper. For more general conditions in which the HVCs can move we should incorporate in Eq. (5) some additional terms. For an HVC moving within the sphere of gravitational influence of the MCs, we should add the term: $\frac{G M_{\mathrm{LMC}}\left(\boldsymbol{r}-\boldsymbol{r}_{\mathrm{LMC}}\right)}{\left(\left|\boldsymbol{r}-\boldsymbol{r}_{\mathrm{LMC}}\right|^{2}+K^{2}\right)^{3 / 2}}$. The term that has into account the interaction of an HVC with the gas of the Galactic disk is given by $-\boldsymbol{F}_{\mathrm{d}}(\boldsymbol{r}, \dot{\boldsymbol{r}})$ (see Paper I, for the meaning of these symbols).

The following phenomenological density profile is used to represent the Milky Way halo of dark matter (see Hernquist 1993)

$\rho_{\mathrm{h}}(r)=\frac{M_{\mathrm{h}}}{2 \pi^{\frac{3}{2}}} \frac{\alpha}{r_{\mathrm{h}}} \frac{\exp \left(-r^{2} / r_{\mathrm{h}}^{2}\right)}{r^{2}+\gamma^{2}}$,

where $M_{\mathrm{h}}$ is the halo mass, $r_{\mathrm{h}}$ is a scale radius, and $\gamma$ is a core radius. The normalization constant $\alpha$ is defined by

$\alpha=1-\sqrt{\pi} q \exp \left[q^{2}[1-\operatorname{erf}(q)]\right]^{-1}$.

Since we can consider that $q=\gamma / r_{\mathrm{h}} \ll 1$, the gravitational potential resulting from Eq. (6) can be written as

$\frac{\partial \phi_{\mathrm{h}}(r)}{\partial r}=\alpha \frac{V_{\mathrm{c}}^{\prime 2}}{r^{2}}\left(-\gamma \arctan \frac{r}{\gamma}+\frac{1}{2} \sqrt{\pi} r_{\mathrm{h}} \operatorname{erf}\left(\frac{r}{r_{\mathrm{h}}}\right)\right)$, where

$V_{\mathrm{c}}^{\prime 2}=\frac{2 G}{\sqrt{\pi}} \frac{M_{\mathrm{h}}}{r_{\mathrm{h}}}$

For simplicity, we adopt $\gamma=0$, and consequently $\alpha=1$. Unlike $\rho_{\mathrm{h}}(r)$, Eq. (8) has not a singularity at $r=0$ when $\gamma$ tends to zero. Values for the two unique parameters that characterize the adopted potential for the halo, $M_{\mathrm{h}}$ and $r_{\mathrm{h}}$, will be estimated in the following.

\subsubsection{Constraints on the mass and scale radius of the Milky Way's halo and on the initial conditions of the HVCs from Conditions 1 and 2}

The linear velocity of rotation due to the halo, denoted here by $V_{\mathrm{h}}$, is obtained from $V_{\mathrm{h}}^{2}=r \frac{\partial \phi_{\mathrm{h}}(r)}{\partial r}=\frac{1}{2} \sqrt{\pi} V_{\mathrm{c}}^{\prime} \frac{r_{\mathrm{h}}}{r} \operatorname{erf}\left(\frac{r}{r_{\mathrm{h}}}\right)$. This relation allows us to obtain $V_{\mathrm{c}}^{\prime}$ as a function of $r_{\mathrm{h}}$ by setting the circular velocity of Galactic rotation in the solar vicinity $V_{\mathrm{h}}=V_{\mathrm{c}}=220 \mathrm{~km} \mathrm{~s}^{-1}$ and $r=r_{\mathrm{s}}=8.5 \mathrm{kpc}$. Note that as $r_{\mathrm{h}} \rightarrow \infty, V_{\mathrm{c}}^{\prime} \rightarrow V_{\mathrm{c}}$ and $\frac{\partial \phi_{\mathrm{h}}(r)}{\partial r} \rightarrow \frac{V_{\mathrm{c}}^{2}}{r}$, the force law used in Paper I. Replacing $V_{\mathrm{c}}^{\prime}$ in Eq. (8), we obtain the force law:

$\boldsymbol{F}_{\mathrm{h}}\left(\boldsymbol{r}, r_{\mathrm{h}}\right)=\frac{1}{r} \frac{\partial \phi_{\mathrm{h}}(r)}{\partial r} \boldsymbol{r}=\frac{V_{\mathrm{h}}^{2} r_{\mathrm{s}}}{r^{3}} \frac{\operatorname{erf}\left(\frac{r}{r_{\mathrm{h}}}\right)}{\operatorname{erf}\left(\frac{r_{\mathrm{s}}}{r_{\mathrm{h}}}\right)} \boldsymbol{r}$

with $r_{\mathrm{h}}$, the scale radius of the halo, as an independent variable or a free parameter which can be in principle fitted through initial and boundary conditions that the trajectories of the MCs should obey. We will trace the orbits of the LMC and SMC back in time, by solving the following equation system:

$\ddot{\boldsymbol{r}}_{\mathrm{S}}+\boldsymbol{F}_{\mathrm{h}}\left(\boldsymbol{r}_{\mathrm{S}}, r_{\mathrm{h}}\right)+\boldsymbol{F}_{\mathrm{L}, \mathrm{S}}\left(\boldsymbol{r}_{\mathrm{L}, \mathrm{S}}\right)=0$

$\ddot{\boldsymbol{r}}_{\mathrm{L}}+\boldsymbol{F}_{\mathrm{h}}\left(\boldsymbol{r}_{\mathrm{L}}, r_{\mathrm{h}}\right)=0$,

where $\boldsymbol{F}_{\mathrm{L}, \mathrm{S}}\left(\boldsymbol{r}_{\mathrm{L}, \mathrm{S}}\right)=\frac{G M_{\mathrm{L}}}{\left(\left|\boldsymbol{r}_{\mathrm{L} S}\right|^{2}+K^{2}\right)^{3 / 2}} \boldsymbol{r}_{\mathrm{L}, \mathrm{S}}$ is the force of the LMC acting on the SMC, and $\boldsymbol{r}_{\mathrm{L}}$ and $\boldsymbol{r}_{\mathrm{S}}$ are the position vectors of the LMC and SMC, respectively, and the distance vector between of the two Clouds $\boldsymbol{r}_{\mathrm{L}, \mathrm{S}}=\boldsymbol{r}_{\mathrm{L}}-\boldsymbol{r}_{\mathrm{S}}, M_{\mathrm{L}}=0.87 \times 10^{10} M_{\odot}$ (van der Marel et al. 2002) is the mass of the LMC and $K=3 \mathrm{kpc}$ (Murai \& Fujimoto 1980) is a parameter of Plummer's potential. The initial conditions of this equation system are the positions and spatial velocities of the both Clouds at the present, $t=0$. Note that we neglected in the above equations the gravitational action of the SMC on the LMC. We adopted $\boldsymbol{r}_{\mathrm{L}}=$ $(-41.30,1.29,-27.43) \mathrm{kpc}$ and $\dot{\boldsymbol{r}}_{\mathrm{L}}=(-218,66,187) \mathrm{km} \mathrm{s}^{-1}$ for the position and velocity vectors of the LMC (see Paper I, for more details on the here adopted parameters). We take the position vector of the SMC corresponding to the distance of $60.3 \mathrm{kpc}$ from the Sun. The observational components from which we obtain the three-dimensional space velocity are the radial velocity $\rho$ and the components $\mu_{\alpha}$ and $\mu_{\delta}$ of the proper motion. Since the proper motion of the SMC is much less well known than that of the LMC, we treat the spatial velocity of the SMC in the following way. We only fixed $\rho$ at the observed value, $148 \mathrm{~km} \mathrm{~s}^{-1}$ and leave the components of the SMC proper motion free. The fact that the Clouds likely joined some hundred $\mathrm{Myr}$ ago (see Paper I, and Sect. 2), give us an extra boundary condition, namely $\boldsymbol{r}_{\mathrm{L}}=\boldsymbol{r}_{\mathrm{S}}$ at the time $t=t_{\mathrm{e}}($ Condition 1$)$. This condition provides three equations that the solutions of the equation system must satisfy with which we can find the unknowns $\mu_{\alpha}$, $\mu_{\delta}$ and $t_{\mathrm{e}}$. With an arbitrarily given $r_{\mathrm{h}}$ we can solve numerically the equation system for the unknowns. Repeating the procedure 


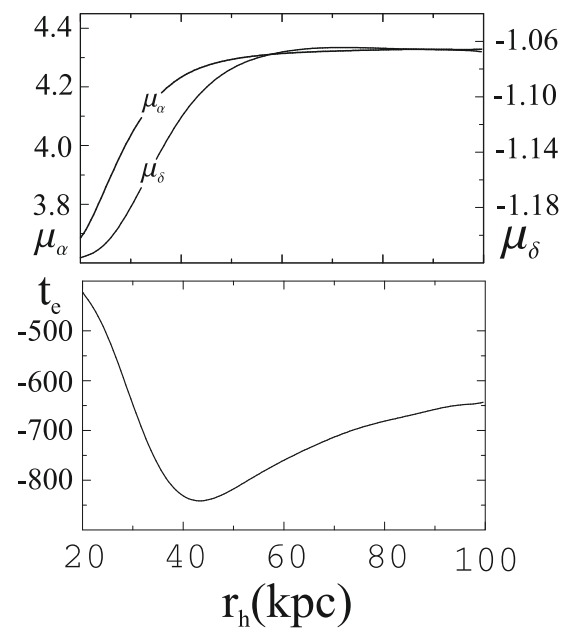

Fig. 3. Components of the theoretical proper motion of the SMC and the encounter time $t_{\mathrm{e}}$ between the LMC and SMC as a function the free parameter $r_{\mathrm{h}}$, the scale radius of the halo. The proper motions and $t_{\mathrm{e}}$ are expressed in mas $\mathrm{yr}^{-1}$ and Myr before the present, respectively.

with different values of $r_{\mathrm{h}}$, we obtain the solutions as a function of this parameter, that is $\mu_{\alpha}\left(r_{\mathrm{h}}\right), \mu_{\delta}\left(r_{\mathrm{h}}\right)$ and $t_{\mathrm{e}}\left(r_{\mathrm{h}}\right)$ (see Fig. 3). To compare with the observations, it is useful to represent the curve $\left(\mu_{\alpha}\left(r_{\mathrm{h}}\right), \mu_{\delta}\left(r_{\mathrm{h}}\right)\right)$ (Fig. 4). Hence, the orbits of the Clouds can be traced as a function of $r_{\mathrm{h}}$ and in particular the position and velocity of the LMC at the time of the collision with the SMC can be calculated (see Fig. 5). With a determined value of $r_{\mathrm{h}}$, we obtain from Fig. 5 part of the initial conditions of the HVCs. The uncertainty in the predicted curve of Fig. 4 results from the uncertainties of the LMC and SMC distances and from the uncertainty of the LMC proper motion. Another uncertainty is the value of the impact parameter, implicitly assumed equal to zero (i.e. a central collision). If the precisions of the observed and theoretical proper motions were sufficiently high, the point representing the observed proper motion should be located on the theoretical curve (see Fig. 4) and would indicate the value of $r_{\mathrm{h}}$.

Figure 4 shows that the predicted proper motions for the $\mathrm{SMC}$ in the range of the possible values for $r_{\mathrm{h}}$ are congruent with the observed proper motion. Indeed, Kroupa \& Bastian (1997), Kallivayalil et al. (2006) obtained $\left(\mu_{\alpha}, \mu_{\delta}\right)=(4.32 \pm 2.9,-1.08 \pm$ $0.8) \mathrm{mas} \mathrm{yr}^{-1}$ and $(3.86 \pm 0.6,-1.17 \pm 0.2)$ mas yr$^{-1}$ respectively. However, there are two factors that do not allow us to find strong constraints on $r_{\mathrm{h}}$. Firstly, the precision of the measured proper motions is insufficient. The appropriate precision of the proper motions should be at least five times higher than the one of the present measurements. Secondly, the predicted proper motions are rather insensitive to $r_{\mathrm{h}}>50 \mathrm{kpc}$. Therefore we will obtain the value of $r_{\mathrm{h}}$ from other procedure, by using the theoretical curves represented by Figs. 3 and 5 and demanding Condition 2. The details of this procedure are given in Appendix A. Here we give in advance the result: $r_{\mathrm{h}}=42 \mathrm{kpc}$ in the case of Model 1 . The theoretical proper motion for $r_{\mathrm{h}}=42 \mathrm{kpc}$ is $\left(\mu_{\alpha}, \mu_{\delta}\right)=$ $(4.25,-1.10)$ mas $^{-1}$, an intermediate value between these two proper motion measurements (see Fig. 4).

Now we show that the mass of the Galaxy corresponding to $r_{\mathrm{h}} \approx 42 \mathrm{kpc}$ is very similar to those obtained by other authors with different procedures. Using $M_{\mathrm{h}}=\frac{V_{\mathrm{h}}^{2} r_{\mathrm{s}}}{G} \operatorname{erf}^{-1}\left(r_{\mathrm{s}} / r_{\mathrm{h}}\right)$, we obtain $M_{\mathrm{h}}=4.24 \times 10^{11} M_{\odot}$. On the other hand, Lynden-Bell et al. (1983), Lin et al. (1995) and Kochanek (1996) obtained $3.9 \times 10^{11}, 5.5 \times 10^{11}$ and $5.1 \times 10^{11} M_{\odot}$ for the mass of the Milky Way, respectively.

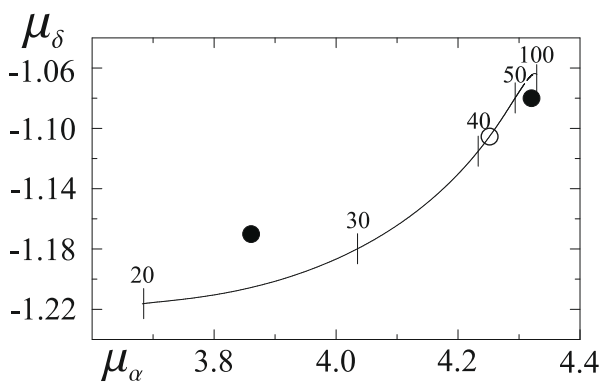

Fig. 4. Theoretical function $\mu_{\delta}$ of $\mu_{\alpha}$ inferred from the corresponding parametric representations of Fig. 3 , The numbers on the curve indicate values of $r_{\mathrm{h}}$ associated with this function. The filled circles represent the measured proper motions and the open circle the adopted one. The

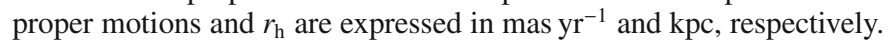

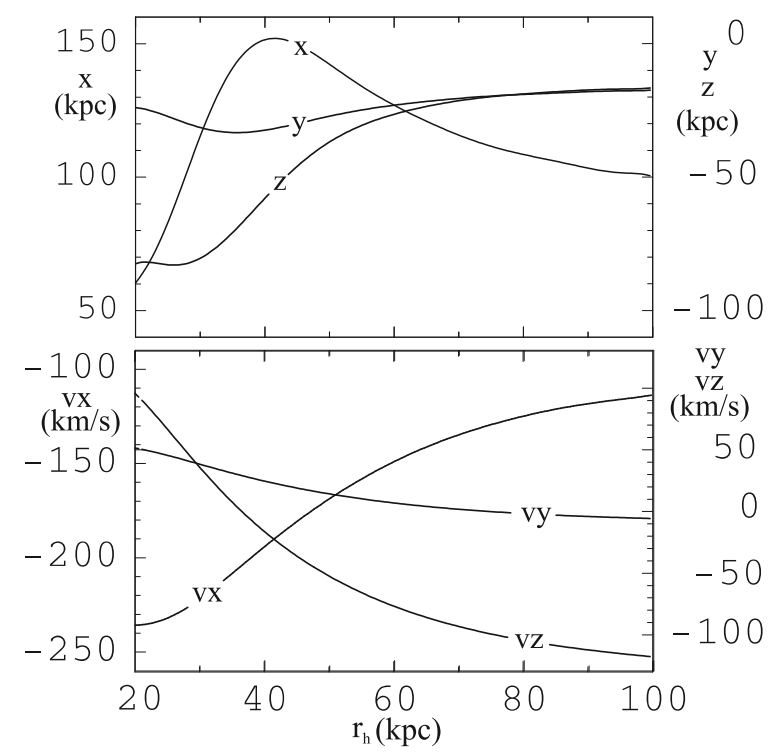

Fig. 5. Position and velocity components of the LMC as a function of $r_{\mathrm{h}}$ at the time of the encounter with the SMC.

The Galactic mass enclosed within the sphere of the radius $r_{\mathrm{o}}$ of the orbits of the MCs is given, in our halo's model, by $M_{\mathrm{h}}\left(r_{\mathrm{o}}\right)=\frac{V_{\mathrm{h}}^{2} r_{\mathrm{s}}}{G} \frac{\operatorname{erf}\left(r_{\mathrm{o}} / r_{\mathrm{h}}\right)}{\operatorname{erf}\left(r_{\mathrm{s}} / r_{\mathrm{h}}\right)}$. Note that $M_{\mathrm{h}}\left(r_{\mathrm{o}}\right) \rightarrow \frac{V_{\mathrm{h}}^{2} r_{\mathrm{s}}}{G}=5.6 \times 10^{11} M_{\odot}$ as $r_{\mathrm{h}} \rightarrow \infty$, which agrees with the mass derived from an isothermal halo distribution. This shows that the Galactic mass that the MCs feel is rather constant for mass distributions with $r_{\mathrm{h}}>$ $50 \mathrm{kpc}$, which explains the insensitivity of the theoretical proper motions shown by Figs. 3 and 4. This also demonstrates that gravitational potential of an isothermal halo distribution, $V_{\mathrm{c}}^{2} \ln r$, is a relatively good approximation of the Galactic potential for $r \leq 50 \mathrm{kpc}$.

With $r_{\mathrm{h}}=42 \mathrm{kpc}$, we obtain that $t_{\mathrm{e}}=-840 \mathrm{Myr}$ (see the lower panel of Fig. 3) and that the position and velocity of the LMC at $t_{\mathrm{e}}$ are

$r_{\mathrm{L}}\left(t_{\mathrm{e}}\right)=(151.96,-31.56,-52.86) \mathrm{kpc}$

$\dot{r}_{\mathrm{L}}\left(t_{\mathrm{e}}\right)=(-188.5,22.5,-24.5) \mathrm{km} \mathrm{s}^{-1}$,

(cf. Fig. 5). They will be used as the initial positions and systemic velocities of the HVCs.

\subsection{Model 2}

The total gravitational potential adopted for Model 2 is $\phi=$ $\phi_{\mathrm{h}}+\phi_{\mathrm{d}}$, where $\phi_{\mathrm{h}}$ and $\phi_{\mathrm{d}}$ denote the halo and disk components, 
respectively. The expression for $\phi_{\mathrm{h}}$ is the same as that adopted for Model 1. The disk potential is represented by the Miyamoto $\&$ Nagai(1975) formula: $\phi_{\mathrm{d}}(x, y, z)=-\frac{G M_{\mathrm{d}}}{\sqrt{x^{2}+y^{2}+\left(a_{\mathrm{d}}+\sqrt{b_{\mathrm{d}}^{2}+z^{2}}\right)^{2}}}$, where $M_{\mathrm{d}}$ is the mass of the Galactic disk, $a_{\mathrm{d}}$ is a scale radius and $b_{\mathrm{d}}$ is a scale height. The variables $x, y, z$ are the components of the position vector $\boldsymbol{r}$. This potential is generated by a density distribution that approximates the luminous components of a disc galaxy, and is amply used in the studies of Galactic dynamics (e.g. Binney \& Tremaine 1987; Besla et al. 2007). The mass distribution of the disk corresponding to this potential is

$$
\rho M(R, z)=\frac{b_{\mathrm{d}}^{2} M_{\mathrm{d}}}{4 \pi} \frac{a_{\mathrm{d}} R^{2}+\left(a_{\mathrm{d}}+3 \sqrt{z^{2}+b_{\mathrm{d}}^{2}}\right)\left(a_{\mathrm{d}}+\sqrt{z^{2}+b_{\mathrm{d}}^{2}}\right)^{2}}{\left[R^{2}+\left(a_{\mathrm{d}}+\sqrt{z^{2}+b_{\mathrm{d}}^{2}}\right)^{2}\right]^{5 / 2}\left(z^{2}+b_{\mathrm{d}}^{2}\right)^{3 / 2}},
$$

where $R^{2}=x^{2}+y^{2}$.

\subsubsection{Constraints on the masses and scale lengths of the Milky Way's halo and disk components and on the initial conditions of the HVCs from Conditions 1 and 2}

Following Kalberla et al. (2007), we adopt the value of $3 \times$ $10^{11} M_{\odot}$ for $M_{\mathrm{d}}$. Since the column density of material at the solar position $r_{\mathrm{s}}$ within $1.1 \mathrm{kpc}, \Sigma_{1.1}$, is $\sim 74 M_{\odot} \mathrm{pc}^{-2}$ (see Kalberla et al. 2007, and references therein), $\int_{-1.1}^{1.1} \rho M\left(r_{\mathrm{s}}, z\right) \mathrm{d} z \equiv \Sigma_{1.1}=$ $74 M_{\odot} \mathrm{pc}^{-2}$, where $\rho M\left(r_{\mathrm{s}}, z\right)$ is given by Eq. (14). Considering $b_{\mathrm{d}}$ as an independent variable, we obtain the corresponding values of $a_{\mathrm{d}}$ that satisfy the $\Sigma_{1.1}$ condition, i.e. $a_{\mathrm{d}}=F\left(b_{\mathrm{d}}\right)$.

The contribution of the disk to the circular velocity of rotation at the solar position is $V_{\mathrm{d}}=\sqrt{\frac{G M_{\mathrm{d}} r_{\mathrm{s}}^{2}}{\left[r_{\mathrm{s}}^{2}+\left(a_{\mathrm{d}}+b_{\mathrm{d}}\right)^{2}\right]^{3 / 2}}}$. Replacing $a_{\mathrm{d}}$ by $F\left(b_{\mathrm{d}}\right)$ and using the relation $V_{\mathrm{c}}^{2}=V_{\mathrm{h}}^{2}+V_{\mathrm{d}}^{2}$, we obtain $V_{\mathrm{h}}$, which here depends on both $r_{\mathrm{h}}$ and $b_{\mathrm{d}}$. Hence Model 2 has two free parameters: $r_{\mathrm{h}}$ and $b_{\mathrm{d}}$. To obtain the motion equations of the Clouds, we should insert the $V_{\mathrm{h}}$ value here calculated into Eq. (9) and add the term $\boldsymbol{F}\left(\boldsymbol{r}, b_{\mathrm{d}}\right)=\nabla \phi_{\mathrm{d}}(x, y, z)$, due to the force field of the disk, to Eqs. (10) and (11). We have defined all elements of Model 2 and can demand that the Clouds orbits satisfy Condition 1. We thus calculated the theoretical proper motion of the SMC (Figs. 6 and 7) and the encounter time $t_{\mathrm{e}}$ as a function of $r_{\mathrm{h}}$ (Lower panel of Fig. 6) as well as the position and velocity of the LMC at $t_{\mathrm{e}}$ (Fig. 8), in analogous way as in Sect. 3.1.1. Here we have fixed the value of $b_{\mathrm{d}}$ at $3520 \mathrm{pc}$, which will be justified in Appendix A.

In order to find the values of $r_{\mathrm{h}}$ and $b_{\mathrm{d}}$, we require the fulfillment of Condition 2. However, we first need to describe the procedure to trace the orbits of the HVCs and to obtain their distances. This will be done in the next 4 sections Here we only give the results of the $r_{\mathrm{h}}$ fitting (see Appendix A). In the case of Model 2, there is no an unique solution for $r_{\mathrm{h}}$, it depends on $b_{\mathrm{d}}$. In other words, we can obtain the solution for $r_{\mathrm{h}}$ as a function of $b_{\mathrm{d}}$. We will adopt $b_{\mathrm{d}}=3520 \mathrm{pc}$ for which $r_{\mathrm{h}}=100 \mathrm{kpc}$, which guarantees Condition 2 and determines reasonable values for the halo mass and the scale lengths of the disk. The value of $3520 \mathrm{pc}$ for $b_{\mathrm{d}}$ implies that $a_{\mathrm{d}}=7614 \mathrm{pc}$. The resulting mass for the halo is $M_{\mathrm{h}}=3 \times 10^{11} M_{\odot}$. Hence the total mass of the Galaxy, $M_{\mathrm{t}}=M_{\mathrm{h}}+M_{\mathrm{d}}$, is $6 \times 10^{11} M_{\odot}$, while from Model 1 $M_{\mathrm{t}}=M_{\mathrm{h}}=4.24 \times 10^{11} M_{\odot}($ see Sect. 3.1 .1$)$. The Galactic mass contained within the sphere of radius $r_{\mathrm{o}}=50 \mathrm{kpc}$ is $4.6 \times 10^{11} M_{\odot}$

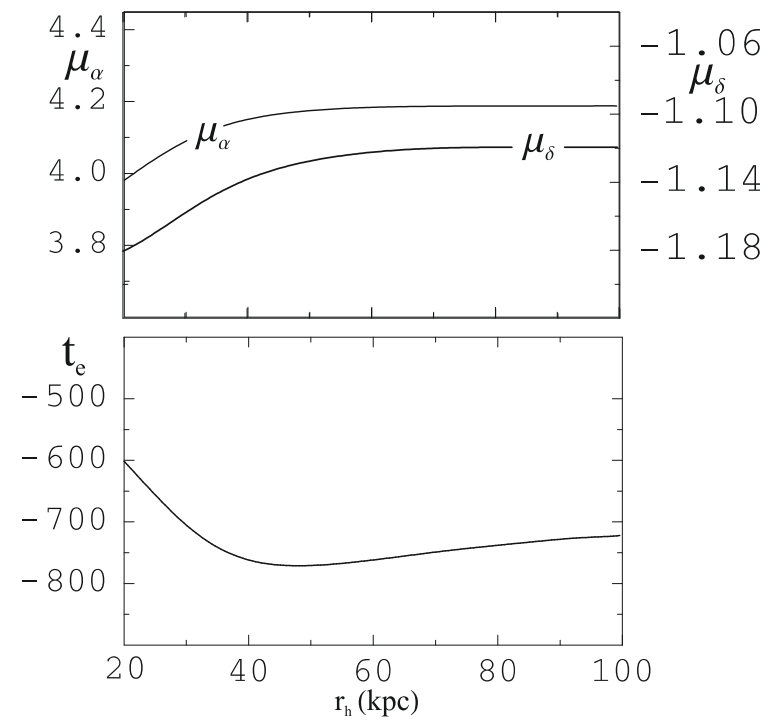

Fig. 6. Same as Fig. 3, but for Model 2 with $b_{\mathrm{d}}=3520$ pc.

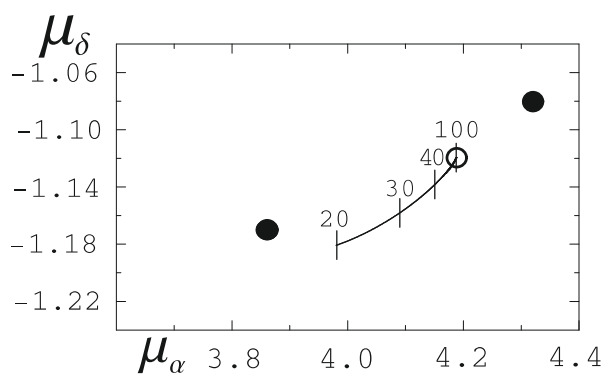

Fig. 7. Same as Fig. 4, but for Model 2.

from Model 2 and $3.9 \times 10^{11} M_{\odot}$ from Model 1 . Note that these masses obtained in our approach agree within the errors with that obtained from the rotation curve to $60 \mathrm{kpc}$ (Xue et al. 2008).

Finally, we obtain the initial conditions of the HVCs as given by Model 2 . With $r_{\mathrm{h}}=100 \mathrm{kpc}$, we find from the functions represented in Figs. 6 and 8 that $t_{\mathrm{e}}=-720 \mathrm{Myr}$ and that the position and velocity of the LMC at $t_{\mathrm{e}}$ are

$\boldsymbol{r}_{\mathrm{L}}\left(t_{\mathrm{e}}\right)=(124.6,-28.2,-48.4) \mathrm{kpc}$
$\dot{\boldsymbol{r}}_{\mathrm{L}}\left(t_{\mathrm{e}}\right)=(-179.3,20.2,-41.1) \mathrm{km} \mathrm{s}^{-1}$

\subsection{Radial velocity versus distance relationships of the HVCs}

We want to solve in this section the following problem. To find the distance $d$ from the Sun to an HVC, by means of our dynamical models and the observable data of the HVC, that is its sky position $(l, b)$ and its radial velocity $\rho$. To do this, we first need to solve the inverse problem, that is if an HVC lies at an assumed distance $d$ from the Sun in the direction $(l, b)$, which radial velocity $\rho$ should this HVC have? In our models, the HVCs were ejected from the MCs when the Clouds meet at the time $t_{\mathrm{e}}=840$ Myr ago according to Model 1 and 720 Myr ago according to Model 2. Therefore the calculation of the orbit followed by a particular HVC that was launched from the position of the LMC at that time (Eqs. (12) and (15) for Models 1 and 2 respectively) and reached the present location at the distance $d$, allows us to obtain the present spatial velocity of this HVC, and 


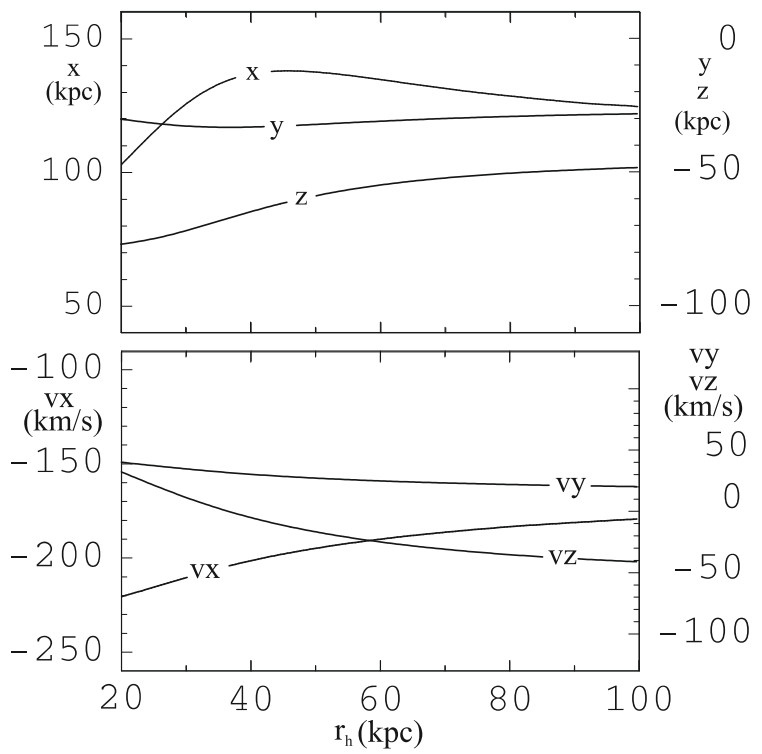

Fig. 8. Same as Fig. 5, but for Model 2 with $b_{\mathrm{d}}=3520$ pc.

hence $\rho$. Assuming several possible distances of an HVC along the direction of the HVC position $(l, b)$ and calculating the corresponding radial velocities $\rho$, we will construct a $\rho-d$ relationship for the direction $(l, b)$. Then, with the observed $\rho$ we obtain through this relationship the real distance of the HVC.

The equation of motion of an HVC subject to the gravitational field of the Galaxy and the LMC, and a drag force $\boldsymbol{F}_{\mathrm{d}}$, experienced by the HVC when moving through the gaseous disk of the Galaxy, can be written as

$\ddot{\boldsymbol{r}}+\boldsymbol{F}_{\mathrm{h}}\left(\boldsymbol{r}, r_{\mathrm{h}}\right)+\boldsymbol{F}_{\mathrm{L}, \mathrm{HVC}}\left(\boldsymbol{r}_{\mathrm{L}, \mathrm{HVC}}\right)+\boldsymbol{F}_{\mathrm{d}}(\boldsymbol{r}, \dot{\boldsymbol{r}})=0$,

where $\boldsymbol{F}_{\mathrm{L}, \mathrm{HVC}}$ is the gravitational force of the LMC exerted on the $\mathrm{HVC}$ and has the same expression as $\boldsymbol{F}_{\mathrm{L}, \mathrm{S}}$, given in the previous section, except that the index $S$ is replaced by HVC. We use for the friction force $\boldsymbol{F}_{\mathrm{d}}$ the expression given in Paper I, only we here consider that the Galactic gaseous layer is warped. In a Galactocentric cylindrical coordinate $\operatorname{system}(R, \phi, z)$, the displacement in $z$ of the mid-plane of the Galactic gaseous layer can be represented by $z_{\mathrm{c}}(R, \phi)$, whose formula we have taken from Binney \& Merrifield (1998). Therefore the density distribution of the gaseous disk of the Galaxy, adopted for the equation of $\boldsymbol{F}_{\mathrm{d}}$, should be now expressed as $n\left(R, z-z_{\mathrm{c}}\right)$. To solve Eq. (17), we need the computation of the LMC orbit, which can be obtained by integrating Eq. (11).

Equation (17) can be solved with the following initial and boundary conditions:1) 840 Myr ago according to Model 1 and 720 Myr ago according to Model 2, chosen as the time origin (i.e. $t=0$, when the two Clouds collided), the position of the HVC coincided with the MC's position (Eqs. (12) and (15) for Models 1 and 2, respectively). 2) At the present (time $t=$ 840 Myr when working with Model 1 and $t=720$ Myr when working with Model 2) the HVC lies at a determined distance $d$ from the Sun in the direction $(l, b)$; with these initial and boundary conditions the motion equations are set up and solved numerically for the two unknowns, the initial and present spatial velocity of the HVC, in the way detailed below.

The present position vector of the HVC, $\boldsymbol{r}^{1}=\left(x^{1}, y^{1}, z^{1}\right)$, is determined by the fixed $(l, b)$ and $d$ through

$x^{1}=d \cos b \sin l$

$y^{1}=r_{\mathrm{s}}-d \cos b \cos l$

$z^{1}=d \sin b$ where $r_{\mathrm{s}}=8.5 \mathrm{kpc}$ is the Galactocentric distance of the Sun. Using an approximate value for the initial velocity vector of the HVC, $\boldsymbol{v}^{0}=\left(\dot{x}^{0}, \dot{y}^{0}, \dot{z}^{0}\right)$, which can be given by Eq. (2) plus the velocity of the LMC at that time, the numerical solution of the motion equations gives us a first approximation to the test position of the HVC and its derivatives. This test position does not generally agree in the first approximation with the position corresponding to the distance we imposed to the HVC. This difference between $\boldsymbol{r}^{1}$ and $\boldsymbol{r}^{t}$, the prescribed position and the calculated one with an approximate initial velocity, is a function of the correction for the initial velocity; and it can be estimated by expanding this function in Taylor series and truncating after the first-order terms. In this way we get the following recursive formulas;

$$
\begin{aligned}
\boldsymbol{r}^{1} & =\boldsymbol{r}_{n}^{\mathrm{t}}+\left(d \dot{x}_{n}^{0} \frac{\partial}{\partial \dot{x}_{n}^{0}}+d \dot{y}_{n}^{0} \frac{\partial}{\partial \dot{y}_{n}^{0}}+d \dot{z}_{n}^{0} \frac{\partial}{\partial \dot{z}_{n}^{0}}\right) \boldsymbol{r}_{n}^{\mathrm{t}} \\
\boldsymbol{v}_{n+1}^{0} & =\boldsymbol{v}_{n}^{0}+d \boldsymbol{v}_{n}^{0} .
\end{aligned}
$$

This linear system with three equations and the three unknowns $\mathrm{d} \boldsymbol{v}_{n}^{0}$, gives the next approximation of the initial velocity, with which the motion equation (Eq. (17)) is again solved, repeating so the procedure. After a sufficient number of iterations normally $\boldsymbol{r}_{n}^{t}$ converges toward $\boldsymbol{r}^{1}$ and we get the solution for $\boldsymbol{v}^{0}$ and $v$ (i.e. $\dot{\boldsymbol{r}}^{1}$ ). Thus we find the LSR radial velocity that an HVC should have if this HVC lies at an assumed distance $d$ from the Sun in the direction $(l, b)$. Repeating the procedure for different distances $d$ of a given direction $(l, b)$, i.e. for various positions equally spaced on the line of sight, we can obtain a relationship between the radial velocity and distance of a particular direction. With the observed radial velocity of an $\mathrm{HVC}$ and the $\rho-d$ relation corresponding to the direction $(l, b)$ of this cloud, we obtain the dynamical distance to the HVC. Figure 9 illustrates the $\rho-d$ relation, given by Models 1 and 2, towards the position of an HVC component. On the basis of a large number of results, we calculated that the mean absolute deviation between the distance predicted by Model 1 and that predicted by Model 2 is $\approx 5 \mathrm{kpc}$. Therefore, both models can be considered quasi-equivalent with respect to the results they give. As Model 1 is the simplest, we will use it in what follows to obtain the HVC distances.

\subsubsection{Determination of distances to Complexes $A$ and $C$}

The $\rho$ to $d$ transformations, derive using using the method described above, is applied in Sect. 4 to the entire set of HVC data, to determine the spatial distribution of the HVC components. To illustrate the virtues and limitations of our method, we show the $\rho-d$ relations given by Model 1 for some sky points of two large systems of HVCs, namely: Complex A and Complex C (Figs. 10 and 11). We distinguish in each curve the part affected by the drag force. This region of the curve is between the point of bifurcation of the curve calculated without the drag force (i.e. $\boldsymbol{F}_{\mathrm{d}}=0$ ) and that in which this force was taken into account (see Figs. 10 and 11). At distances lower than the distance of the bifurcation point $d_{\mathrm{b}}$, the HVC is braked by the Galactic gas, dismissing the absolute value of the velocity. Even though the velocity-distance relation for $d>d_{\mathrm{b}}$ is not exactly linear, this part of the curve is well fitted by Eq. (4) derived from the kinematical model. The differences between the kinematical and dynamical distances are about $8 \mathrm{kpc}$. There is a fundamental difference, however, between kinematic velocity-distance and dynamical relations: the second relation is often a two-valued function, which, in certain velocity ranges, provides two possible distances for a given radial velocity that is, in the case of Complex $A$ and $C$, the 


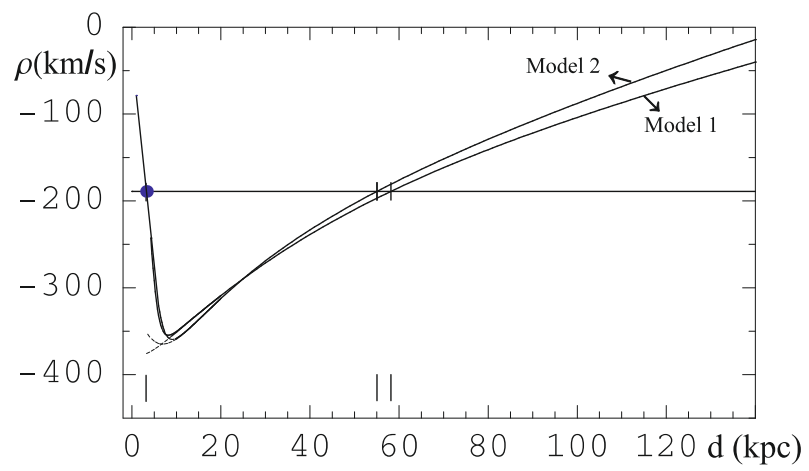

Fig. 9. Comparison of results of Model 1 with Model 2. The $\rho-d$ relationships from Models 1 and 2 for the position $(l, b)=\left(139^{\circ}, 28^{\circ}\right)$ of Complex A are represented. The horizontal line corresponds to the radial velocity of the HVC component, $\rho=-189 \mathrm{~km} \mathrm{~s}^{-1}$. The short vertical lines crossing the curves indicate the points whose ordinates equal to the radial velocity of the HVC component, and hence their abscissas give the respective solutions for the far distance of this HVC component (58 kpc from Model 1 and $55 \mathrm{kpc}$ from Model 2). Similarly, the big dot shows the solutions for near distance of the HVC component, which are almost coincident $(\approx 3 \mathrm{kpc})$. The broken lines indicate the part of the curve that was calculated without drag forces and modified by the introduction of this resistive force.

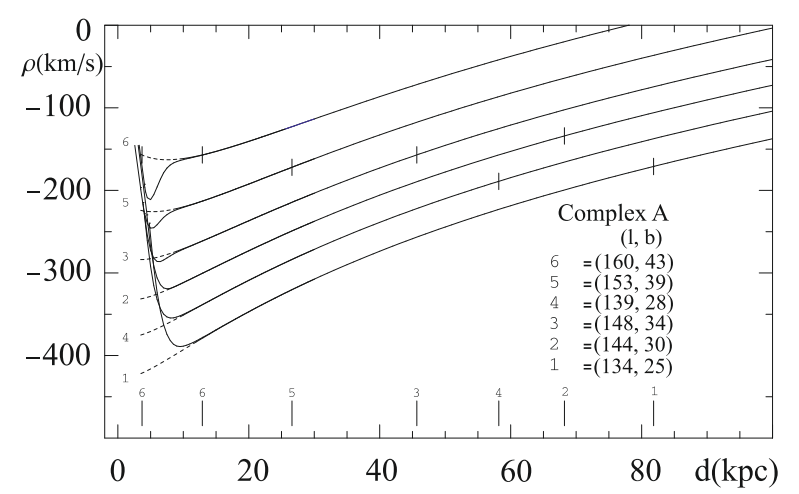

Fig. 10. The $\rho-d$ relationships for various sky positions on Complex A. The radial velocity $\rho$ is referred to the LSR and the distance is from the Sun. The labels of 1 to 6 on the curves identify their respective Galactic co-ordinates in the attached table and the far-distance solutions on the $d$-axis. The broken lines indicate the part of the curve that was calculated without drag forces and modified by the introduction of this resistive force. The small vertical line crossing each curve indicates the point on the curve whose ordinate is equal to the LSR radial velocity of the corresponding component of Complex A, and hence its abscissa is the solution for the distance to this component of Complex A.

closest distance $<d_{\mathrm{b}}$. The closest distances for both Complexes are within a narrow range between 4 and $6 \mathrm{kpc}$. In this case, the different gas concentrations of each complex have almost identical initial velocities, that is they have traveled together. Hence, the dispersion of their observed velocities is due to a recent action by the drag force that decelerated differentially the clouds. In contrast, the furthest distances range from 10 to $80 \mathrm{kpc}$ for Complex A and from 10 to $150 \mathrm{kpc}$ for Complex C. The dispersion of the present velocities, in this case, reflects the dispersion of initial velocities corresponding to the furthest distances, which explains the large scatter for both complexes.

The only part of Complex A for which it has been possible to determine optically a secure lower and upper limit to the distance, is the upper section of Chain A (van Woerden et al. 1999). In this direction $\left(l=160^{\circ} .4, b=43^{\circ} .3\right)$, Complex A lies between 4 and $11 \mathrm{kpc}$, as predicted by our model (see curve 6

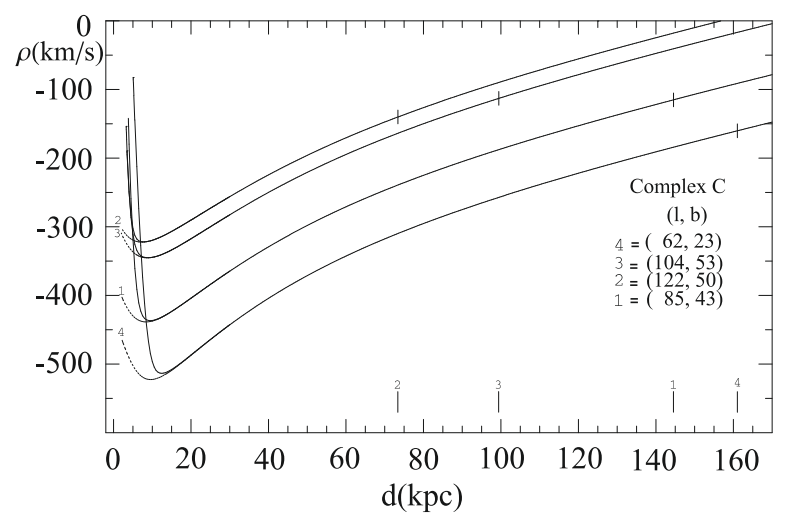

Fig. 11. Same as Fig. 10, but for Complex C.

of Fig. 10). This region has the particularity that its near and far distances are almost coincident within the errors. This fact does not enable the distance ambiguities for the remainder of the positions of Complex A to be resolved. In other words, we require distance estimates of positions inside Complex $\mathrm{A}$, for which the separations between the near and far distances are large. For Complex C, we know of only a lower limit to the distance, $d>5 \mathrm{kpc}$. When this paper was being reviewed, we knew from Wakker et al. (2007) that the distance to Complex C was estimated to be in the range 3.7-11.2 kpc; Thom et al. (2007) also estimate a distance in the range $10-15 \mathrm{kpc}$. The agreements between these measured distances and the corresponding values estimated on the basis of our dynamical model, provide support to the model and its adopted parameters such as $r_{\mathrm{h}}$.

\section{Results of the dynamical model}

Since both dynamical models provide similar results on the overall space distribution of the HVCs, we focus our attention hereafter on the results of Model 1, for which we fitted only one free parameter. Our objective is to calculate the $\rho-d$ relations for the 10940 sky positions of Wakker's list toward which HVC emissions were detected. To save computational time and facilitate the convergence of the solutions of Eq. (17), we simplify this equation by neglecting the term $\boldsymbol{F}_{\mathrm{L}, \mathrm{HVC}}$. We note that the exact solution of the present spatial velocity of an HVC is unaffected by the omission of this term. In other words, we obtain similar $\rho-d$ relations, solving Eq. (17) with or without this term. However, the solution for the initial velocity of the HVC given by the incomplete equation should be interpreted in a different way from the original definition. In our simplification, we consider that the HVC moves only under the action of the gravitational field of the halo. Hence, the initial velocity of the HVC, in this condition, which wedenote $v_{i}^{\prime}$, must satisfy the conservation law of the total energy given by $v_{\mathrm{i}}^{\prime 2}-v(\boldsymbol{r})^{2}=2\left(\phi_{\mathrm{h}}\left(\boldsymbol{r}_{\mathrm{L}}\left(t_{\mathrm{e}}\right)\right)-\phi_{\mathrm{h}}(\boldsymbol{r})\right)$. In the calculation of the actual initial velocity of the $\mathrm{HVC}$, we should consider the gravitational field of the LMC, $\phi_{\mathrm{L}}$, and hence $v_{\mathrm{i}}^{2}-v(\boldsymbol{r})^{2}=2\left(\phi_{\mathrm{h}}\left(\boldsymbol{r}_{\mathrm{L}}\left(t_{\mathrm{e}}\right)\right)-\phi_{\mathrm{h}}(\boldsymbol{r})\right)-2\left(\phi_{\mathrm{L}}(0)-\phi_{\mathrm{L}}\left(\boldsymbol{r}-\boldsymbol{r}_{\mathrm{L}}\right)\right)$. The velocity of the HVC at $\boldsymbol{r} v(\boldsymbol{r})$ has a similar value in both equations, since it must fulfill the boundary conditions. Eliminating $v(\boldsymbol{r})$ from both equations, $v_{\mathrm{i}}^{2}-v_{\mathrm{i}}^{\prime 2}=2\left(\phi_{\mathrm{L}}(0)-\phi_{\mathrm{L}}\left(\boldsymbol{r}-\boldsymbol{r}_{\mathrm{L}}\right)\right)$. This equation can be rewritten as $\Delta v_{\mathrm{i}}^{2}+2 v_{\mathrm{i}}^{\prime} \Delta v_{\mathrm{i}}=\frac{G M_{\mathrm{L}}}{K}-\frac{G M_{\mathrm{L}}}{\sqrt{\left|\boldsymbol{r}-\boldsymbol{r}_{\mathrm{L}}\right|^{2}-K^{2}}}$, where $\Delta v_{\mathrm{i}}=v_{\mathrm{i}}-v_{\mathrm{i}}$, the correction for the initial velocity of the HVC. The value of $v_{i}$ is provided by the solution of the simplified Eq. (17), and $v_{\mathrm{i}}$ is obtained by solving the above quadratic equation for $\Delta v_{i}$, with the condition that $\Delta v_{\mathrm{i}}>0$. 
The simplest and quickest way to solve Eq. (17) and consequently obtain the $\rho-d$ relations is to consider that the drag force $F_{\mathrm{d}}=0$. This enables significant quantities of data to be processed and results can be compared with those obtained from the kinematic model. Since we assume that the halo is devoid of gas and that the drag force acts only within the Galactic disk, the condition $F_{\mathrm{d}}=0$ is satisfied for a significant part of the distance range of the $\rho-d$ relations, for the purpose of these calculations. Although the gas density $n_{\mathrm{h}}$ of the halo, before the injection of the HVCs, was probably low, $n_{\mathrm{h}}$ was not zero. The local intergalactic medium has low volume densities, $10^{-6}-10^{-4} \mathrm{~cm}^{-3}$ (Richter 2006). If $n_{\mathrm{h}} \approx 10^{-6}$ atoms $\mathrm{cm}^{-3}$ (i.e. 1 atom $\mathrm{m}^{-3}$ ), the velocity variation $\Delta v$ of an $\mathrm{HVC}$ due to the action of halo friction, during the period $T=840 \mathrm{Myr}$, is $\approx 10$ per cent of its initial velocity $v_{\mathrm{i}}$. These figures can be obtained from $\Delta v \approx F_{\mathrm{d}} T=-\frac{n_{\mathrm{h}}}{N_{\mathrm{H}}} v_{\mathrm{i}}^{2} T$, where $N_{\mathrm{H}}=10^{19}$ atoms cm $\mathrm{cm}^{-2}$ and $v_{\mathrm{i}}=300 \mathrm{~km} \mathrm{~s}^{-1}$ (see Paper I). The mean gas density of the halo regions containing HVCs would be ten times greater than the background gas density $n_{\mathrm{h}}$ that we provide as an example (i.e. $\sim 10$ atoms $\mathrm{m}^{-3}$, see Sect. 6). In the following, we study first the case of a null drag force and then incorporate this force into our calculations to study the main complexes of HVCs.

\subsection{Analysis of the $\rho-d$ relations calculated without drag forces}

Although these $\rho-d$ relations are valid strictly only for distances greater than the distance to the edge of the Galactic disk in the considered direction, we represent them in the complete range of distances. From the $\rho-d$ relations calculated for a regular grid of positions $(l, b)$ over the whole celestial sphere, we represent $\rho$ as a function of the Galactic longitude $l$ and the distance $d$, for a fixed Galactic latitude $b$, that is $\rho(l, d)$ at $b=$ constant (see Fig. 12). We calculated the $\rho-d$ relation for each observed HVC to determine its dynamical distance, that is we obtained the $\rho-d$ curves for the 10940 positions of observed HVC components catalogued by Wakker. Depending on whether the line defined by the observed $\rho=$ constant of an HVC crosses the $\rho-d$ curve of this particular HVC, we have one of the following cases: (i) there is no solution for the dynamical distance of the HVC; (ii) there is a unique solution for the dynamical distance of the HVC; (iii) there are two solutions for the dynamical distance of the HVC: the near distance and the far distance.

The position $(l, d)$ of each observed HVC component (i.e. cloud element), in the cases (ii) or (iii), in a certain latitude range centered on $b$, is plotted on the $\rho(l, d)$ diagram at $b$ (see Fig. 12). In the first and second Galactic quadrants, apart from between $l=0^{\circ}$ and $30^{\circ}$, the $\rho-d$ relations are single-value functions and most HVC components in these two quadrants belong to case (ii) (see Figs. 10 and 11). However, when considering the drag force (see Figs. 10 and 11 and the next subsection), the $\rho-d$ relations of these two quadrants become two-value functions and the solutions of the distances of the HVCs, plotted in these $\rho(l, d)$ diagrams, correspond to the "far" distances.

In the third and fourth quadrants, the $\rho-d$ curves are twovalue functions and the represented HVCs have two possible distances. However, we show that the "near" distances, which are represented using the minus signs, should be discarded, which removes the distance ambiguities. The distances corresponding to the minimum values of $\rho$ in the $\rho-d$ curves of these two last quadrants are close to the distance to the edge of the Galactic disk in those directions, which we refered to as "distances of bifurcation" in the previous section. If we include the drag forces, the $\rho-d$ curves of this region therefore bend down at the bifurcation distances, converting the two-value functions into one-value functions.

The majority of the HVCs belonging to the case (i) are in the third and fourth quadrants and have velocities lower that the minimum values of $\rho$ in the $\rho-d$ curves, and therefore cannot be represented in the diagrams. To explain the kinematics of this group, we must consider the drag forces. These HVCs, which we refer to as population W, were braked by the gas of the Galactic disk and their distances should be lower than $30 \mathrm{kpc}$. Further details are provide in the next subsection.

Using the distances to the observed HVC components calculated using the method described above, we derive the spatial distribution of the HVCs shown in Fig. 13. We note the close correspondence between the distribution resulting from the application of formula (4) of the kinematic model (Fig. 2) and the distribution obtained from the dynamic model without drag forces. Expressing the initial velocity of an HVC as the sum of a peculiar velocity and the adopted systematical velocity, that is the LMC velocity at the time $T=-840 \mathrm{Myr}$, Fig. 14 shows the distribution of the peculiar initial velocities of the HVCs. By comparing Figs. 13 and 14, we note that the spatial and initial velocity distributions of the HVCs are approximately homologous, which indicates that the distance traversed by an $\mathrm{HVC}$ is almost proportional to the initial velocity of the $\mathrm{HVC}$ and that the kinematical distance to the HVC, which is given by Eq. (4), is a good approximation to the HVC distance. The difference between the kinematic and dynamic distance is typically about $8 \mathrm{kpc}$. The distribution in Fig. 13 of data for the third and fourth Galactic quadrants is unique, although a population of HVCs (Population W), braked by the gas of the Galactic disk, is missing. When considering drag forces due to the Galactic gaseous disk, the distribution for the first and second Galactic quadrants is not unique, since each position in Fig. 13, represented in these two quadrants, has the alternative of a near position (i.e. within the Galactic disk).

According to these far-distance solutions, Complexes A and $\mathrm{C}$ and the remainder of the HVC complexes of the first and second quadrants, such as Complexes M, H, ACHVC and L (see Table 3 of Wakker \& van Woerden 1991), apart from the clouds of the classic Magellanic Stream (MS) below $b=-15^{\circ}$, are part of a gigantic structure that has the shape of a ring or shell (see Figs. 12 and 13). Since these HVC complexes have similar spatial and kinematic distribution, we call them Population A-C.

\subsection{Analysis of the $\rho-d$ relations calculated including drag forces}

From Figs. 10 and 11, we see that the near distances to Complexes $\mathrm{A}$ and $\mathrm{C}$ are approximately $6 \mathrm{kpc}$. Hence, these neardistance solutions to these two prominent complexes, are assigned to the entire Population A-C. Figures 15 and 16 show the orbits of the components of Complexes $\mathrm{A}$ and $\mathrm{C}$ analyzed in Figs. 10 and 11, which correspond to their motion in the past 100 Myr following the strong interaction of the HVCs with gas in the Galactic disk. These orbits should be considered only qualitatively because friction law adopted, and the density and velocity distribution of the interstellar gas in the Galactic disk on which the friction depends, are highly idealized. We found that the initial peculiar velocities of Complexes A and $\mathrm{C}$ are $(\mathrm{VXi}, \mathrm{VYi}, \mathrm{VZi})=(-185 \pm 1,100 \pm 2,65 \pm 1) \mathrm{km} \mathrm{s}^{-1}$ and $(-187 \pm 1,111 \pm 2,74 \pm 3) \mathrm{km} \mathrm{s}^{-1}$, respectively. The similarity of these initial velocities, or initial positions in phase space, of Population $\mathrm{A}-\mathrm{C}$ indicates that this group of $\mathrm{HVCs}$ 

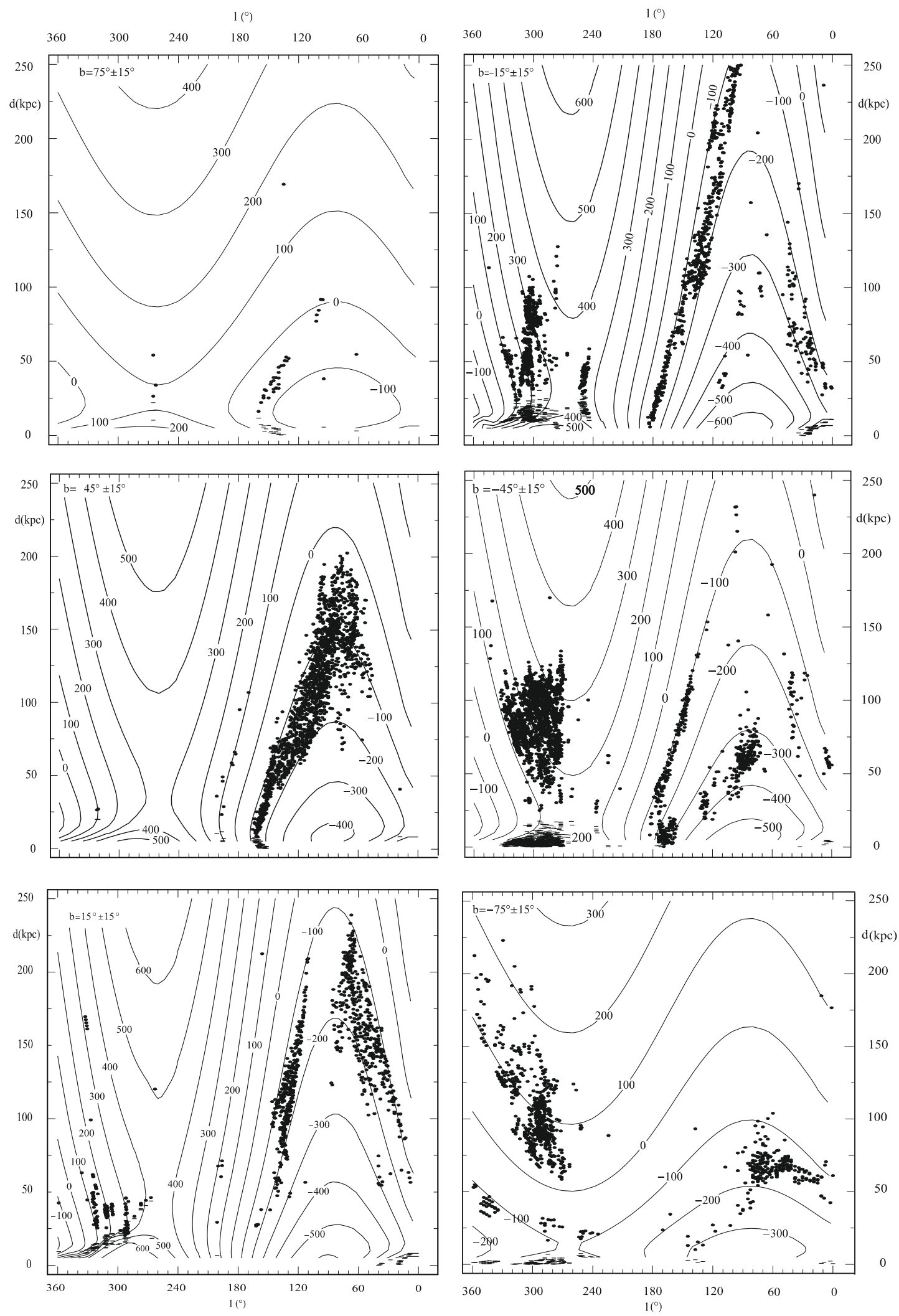

Fig. 12. Contours of LSR radial velocity plotted as a function of Galactic longitude and distance from the Sun, for a given interval of Galactic latitudes. The localizations of the HVC components at the far distances (dots) and at the near distances (minus symbol) are shown. The calculations were made using Model 1 without including drag forces.

formed a relatively compact structure, until its penetration into the Galactic gas layer caused its fragmentation and dispersion (see Figs. 15 and 16). The density structure of both the Galactic layer and HVCs must have important effects on the trajectory of these HVCs. The envelopes of the HVCs interacting with the Galactic layer are decelerated more rapidly than their denser nuclei, which tend to retain a "dynamical memory" of their origin. This would explain naturally the systematic velocity shifts 


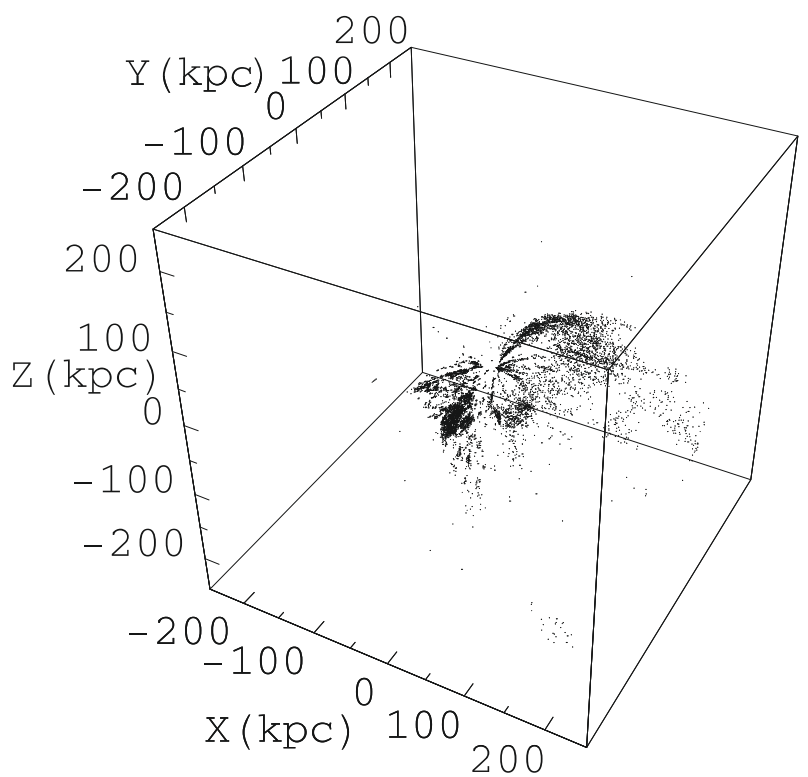

Fig. 13. Spatial distribution of the HVC components according to the dynamic model (Model 1), considering that the HVCs have not interacted with the gas of the Galactic disk. The positions are referred to the Galactocentric coordinate system $(X, Y, Z)$ defined in the text.

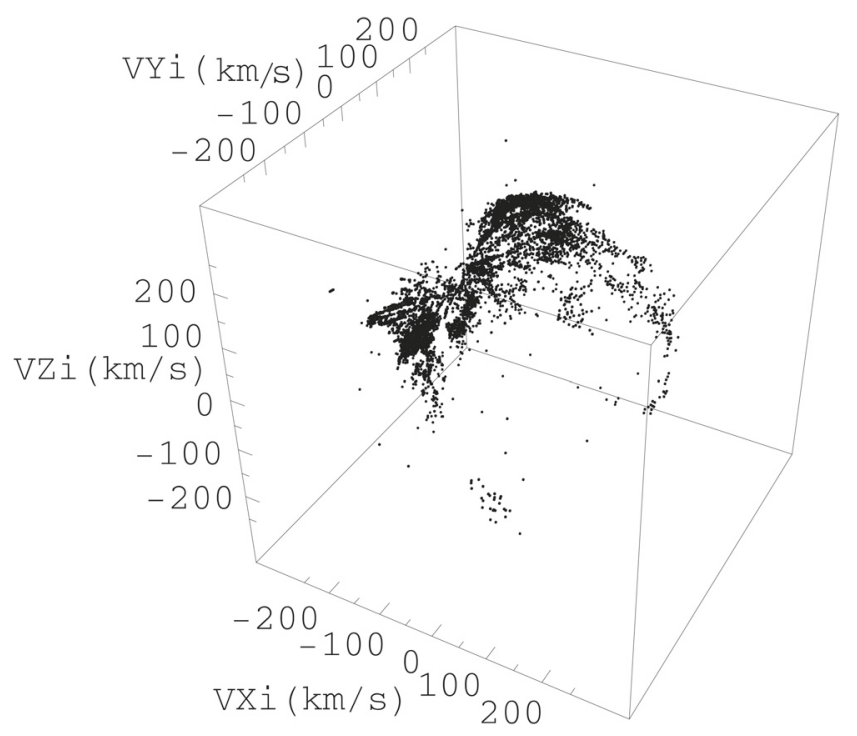

Fig. 14. Distribution of the peculiar initial velocities of the HVC components.

observed between the envelopes and nuclei of Complexes A and C (Kalberla \& Haud 2006). These complex processes can be investigated by means of hydrodynamical simulations of the HVC-Galactic disk collision (Tenorio-Tagle 1980, 1981; Comerón \& Torra 1992; Santillán et al. 1999), for which our models can provide initial conditions. The entry velocities of HVCs into the Galactic layer predicted by our models are much greater than those predicted by a model of Galactic infall (Benjamin \& Danly 1997). Detailed simulations should allow us to test the effects of this difference.

The near-distance solutions for Population A-C imply certainly that these HVCs are interacting with the Galactic disk, and the dynamical models explain coherently the present velocities of these HVCs after strong deceleration. We emphasize that this is not a weakness of our models, but rather a strength. Population $\mathrm{A}-\mathrm{C}$ form a vast system that has been interacting

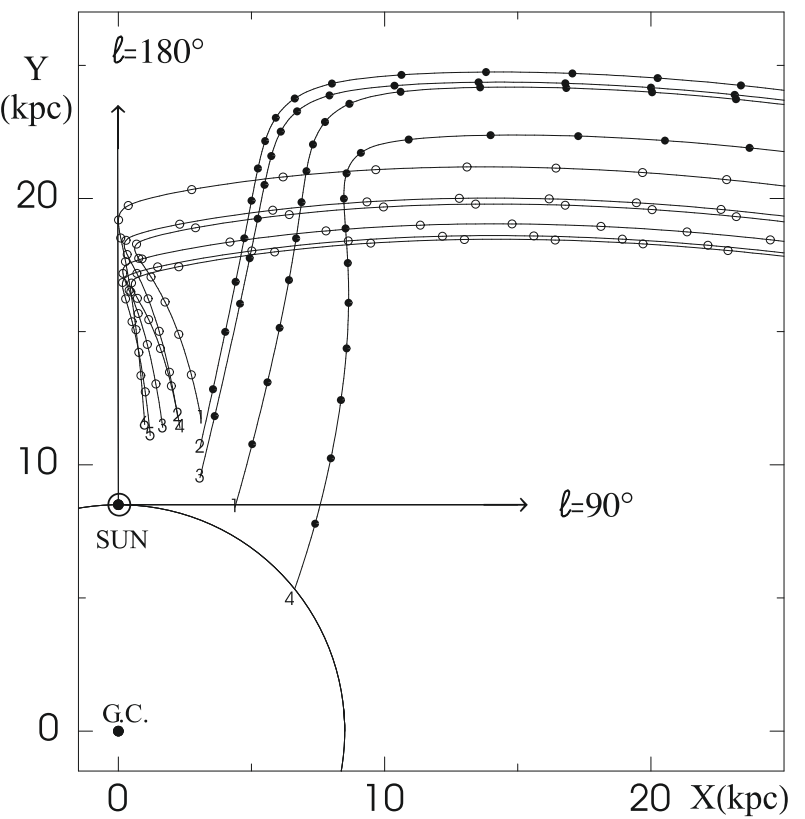

Fig. 15. Orbits of components of Complex A (line with open circles) and of Complex C (line with filled circles), projected onto the $X-Y$ plane, according to the near-distance solutions obtained from $\rho-d$ relationships of Figs. 10 and 11. The labels from 1 to 6 at the ends of the orbits (i.e. present positions of these HVCs) correspond to the same labels of Figs. 10 and 11, identifying the HVC components. The circles on the orbits mark time every 10 Myr.

with the low-density edge of the Galactic disk, for a relatively short time, and whose dynamical memory and traces have not yet been destroyed. Hence, we are able not only to identify its dynamical origin, but also explain the formation of certain Galactic structures as a consequence of this HVC-Galactic disc interaction.

If the HVCs of Population A-C are located at the "near" distances, the formation of HI large structures in the Galaxy, such as the anticenter shell (Mirabel 1982; Heiles 1984; Tamanaha 1996) and the Low-Latitude Intermediate-Velocity Arch (Wesselius \& Fejes 1973; Kuntz \& Danly 1996; Wakker et al. 1998), can be easily explained in terms of a stream of HVCs in different states of collision with the Galactic disk. We mention that the anticenter HVCs, the Giovanelli stream, and the Cohen stream have a kinematical pattern similar to that of Population A-C, and hence could be southern components of the same stream of HVCs. Wakker et al. (2008) derived distance brackets of 5 to $11.7 \mathrm{kpc}$ for the Cohen stream. All this speaks strongly in favour of the near-distance solutions for Population A-C.

The anticenter region delimited by $196^{\circ} \leq l \leq 160^{\circ}$ and $-50^{\circ} \leq b \leq 10^{\circ}$, could serve as an example for conditions of entry of the HVCs into the Galactic layer. This sky region contains the anticenter chain of HVCs (ACHVCs) labeled ACI, ACII, ACII, HVC168-43-280, Cohen stream, and Giovanelli stream (see Fig. 9 of Wakker 2001a). The properties of this region could be explained by a sequence of collisions of HVCs with the Galactic layer, in which the leading clouds of the HVC chain transfer their masses and kinetic energies completely to the surrounding disk gas and generate a large low-density cavity whose walls are made up of low- and intermediate-velocity HI filaments. This is a distinctive HI structure, which is usually referred to as the anticenter shell (Heiles 1984). The trailing HVCs that penetrated into the previously created cavity, which interact with 


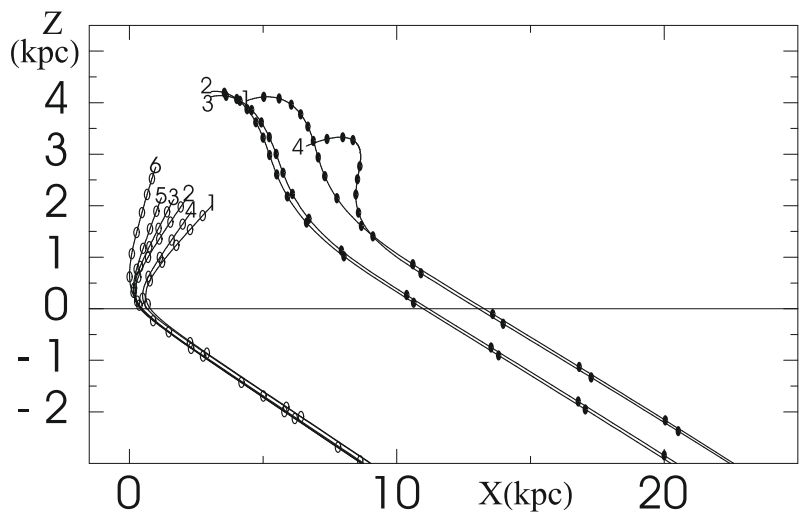

Fig. 16. Same as Fig. 15 but for the projection onto the $X-Z$ plane.

the inner low-density gas, are now observed as ACHVCs. The HVCs at the entry to the cavity, which is at position $l \sim 170^{\circ}$ and $b \sim-30^{\circ}$ (see Fig. 7 of Tamanaha 1996), have high velocities $\left(\sim-300 \mathrm{~km} \mathrm{~s}^{-1}\right)$ and must not have experienced significant braking. These high velocities can be explained by the models without including drag forces (see Fig. 12), but ACHVCs lying at $l>175^{\circ}$ were perturbed by an interaction with low velocity gas. Tamanaha $(1995,1996)$ showed that the incoming ACHVCs came from below the Galactic plane, in accordance with our model predictions (see Paper I). Therefore, the magnitude and direction of the entry velocities of the HVCs, which are provided in this case by our models, appear agree well with the observational data.

To estimate the distance to the so-called Population $\mathrm{W}$ in the previous section, a group of positive-velocity clouds lying in the third and fourth Galactic quadrants, is required to obtain its $\rho-d$ relations calculated including drag forces. These clouds, which were first reported by Wannier et al. (1972), were divided into four separate complexes WA-WD (Wakker \& van Woerden 1991). When the drag force is relatively large, the convergence of the solutions is slow and complicated. We therefore obtained the $\rho-d$ relations for only a few components of the Wannier clouds (Fig. 17). From these $\rho-d$ relationships, we find that Population W lies in the outskirts of the Galaxy at a distance of approximately $15 \mathrm{kpc}$ from the Sun. We note that there is no degeneracy in the value of the distance. In Figs. 18 and 19, we show the orbits corresponding to the solutions of Fig. 17. These theoretical distances for Population $\mathrm{W}$ are consistent with limits derived using optical observations of background stars (Sembach et al. 1991; Thom et al. 2006).

According to the predictions of our theoretical model, the HVCs can be grouped into three populations: A-C, W, and the population of the Magellanic Stream, MS. We found that the spatial distribution derived using the dynamical model for the HVCs in the third and fourth quadrants, which corresponds to population $\mathrm{W}$ and part of population MS (i.e. part of the classic MS and its leading arm), is unique. Although we have two possible values of distances for the HVCs in the first and second quadrants, we choose the far distances, after applying continuity criteria, as the solutions for the HVCs of population MS that are in the first and second quadrants i.e. part of the classic MS. Therefore, only Population $\mathrm{A}-\mathrm{C}$ has an ambiguous value of the distance.

In Fig. 20, the spatial distribution corresponding to the three populations of HVCs is represented by the spatial positions of HI components of Population MS, and ellipses delineated the entire location of Population $\mathrm{W}$ and the near location of Population A-C. The number of HVCs in the original

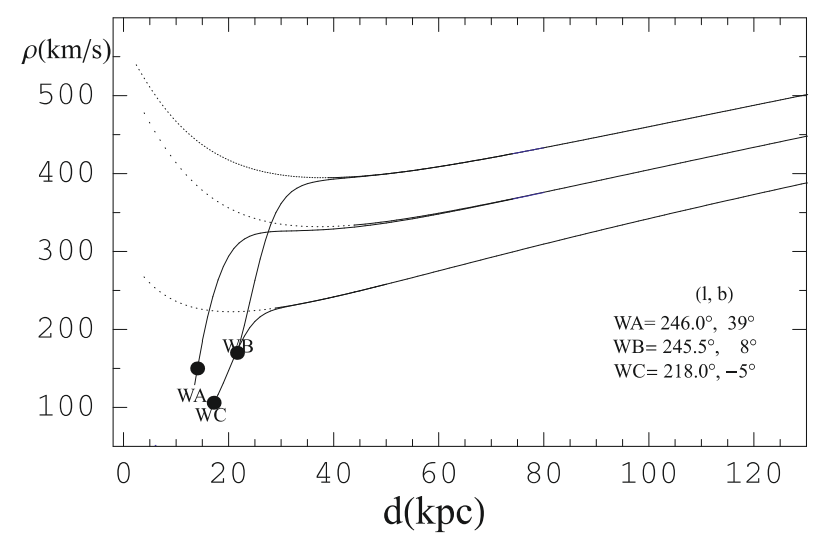

Fig. 17. The $\rho-d$ relationships for three sky positions of HVC components of Population W. The radial velocity $\rho$ is referred to the LSR and the distance is from the Sun. The labels WA, WB and WC on the curves identify their respective Galactic co-ordinates in the attached table. The broken lines indicate the part of the curve that was calculated without drag forces and modified by the introduction of this resistive force. The filled circle on each curve indicates the point on the curve whose ordinate is equal to the LSR radial velocity of the corresponding HVC component, and hence its abscissa is the solution for the distance to this HVC component.

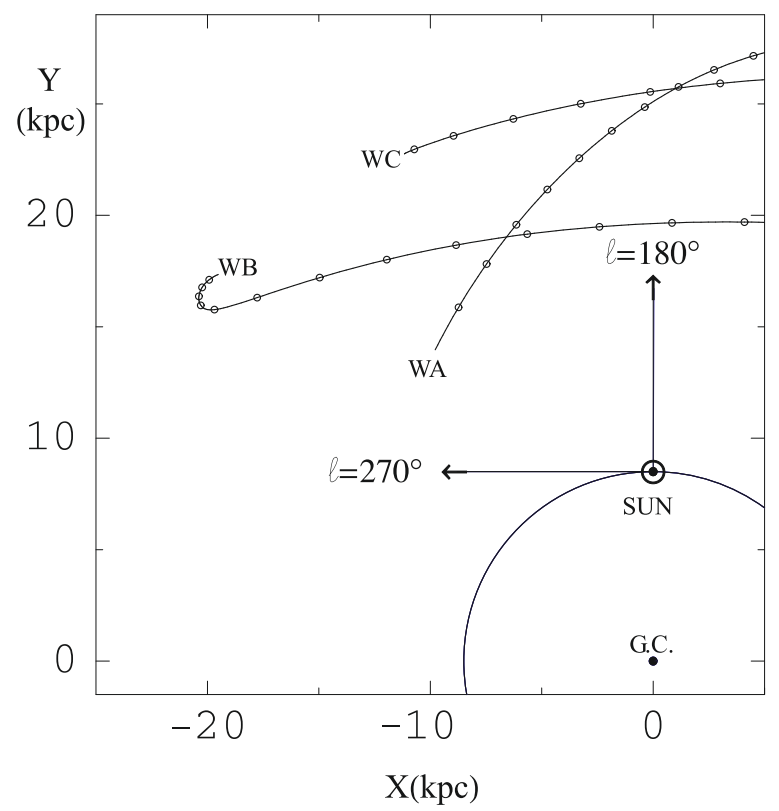

Fig. 18. Orbits of components of Population W, projected onto the $X-$ $Y$ plane, corresponding to $\rho-d$ relationships of Fig. 17. The labels WA, WB and WC at the ends of the orbits (i.e. present positions of these HVCs) correspond to the same labels of Fig. 17, identifying the HVC components. The circles on the orbits mark time every $10 \mathrm{Myr}$.

population larger than that represented in Figs. 13 and 20 since a part of the HVCs was absorbed by the Galactic gas layer and the dynamical history of a particular HVC that was subjected to this braking process, cannot in principle be reconstructed. In the simulation developed in Paper I, we showed that this group of HVCs in strong interaction with the Galactic gas layer was likely to be responsible for the generation of the Galactic warp (see Paper I; and Momany et al. 2006).

A synopsis of the main results is given in Table 1. Columns 5 and 6 of Table 1 contain the total $\mathrm{HI}$ mass $\left(M_{\mathrm{H}}\right)$ and original kinetic energy $\left(E_{\mathrm{K}}\right.$ with which the HVCs were expelled from the Magellanic Clouds) of each population. For the 
Table 1. Characteristics of the HVC populations.

\begin{tabular}{|c|c|c|c|c|c|}
\hline Population & Sky region & Radial velocities $\left(\mathrm{km} \mathrm{s}^{-1}\right)$ & Distances (kpc) & HI mass $\left(M_{\odot}\right)$ & Kinetic energy (erg) \\
\hline MS & $\begin{aligned} 0 & <l<100 \text { for } b<-15 \\
\text { and } 180 & <l<360 \text { for }-90<b<25\end{aligned}$ & -400 to 400 & 20 to 250 & $3.34 \times 10^{8}$ & $2.4 \times 10^{56}$ \\
\hline W & $200<l<320,-50<b<50$ & 90 to 345 & $\sim 15$ & $7 \times 10^{6}$ & $3.5 \times 10^{54}$ \\
\hline \multirow[t]{2}{*}{$\mathrm{A}-\mathrm{C}$} & $\begin{array}{l}\quad 0<l<180 \text { for } b>-15 \\
\text { and } 100<l<180 \text { for } b<-15\end{array}$ & -460 to 150 & 5 to 250 (far distances) & $5.34 \times 10^{8}$ & $2.9 \times 10^{56}$ \\
\hline & & & $\sim 6$ (near distances) & $1 \times 10^{6}$ & $5.5 \times 10^{53}$ \\
\hline
\end{tabular}

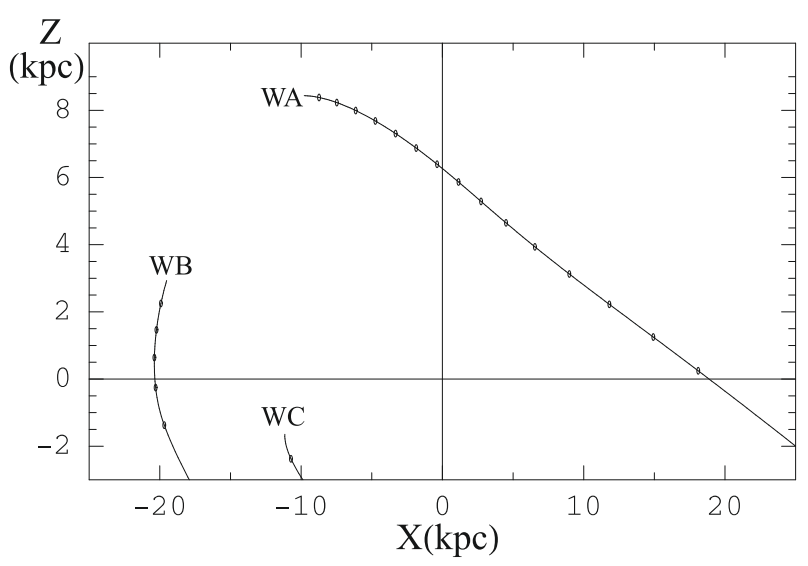

Fig. 19. Same as Fig. 18, but for the projection onto the $(X-Z)$ plane.

calculation of the masses, we refer the reader to Paper I. The kinetic energy $E_{\mathrm{K}}$ is calculated by means of $\frac{1}{2} \sum m_{\mathrm{H}} v_{\mathrm{p}}^{2}$, where $m_{\mathrm{H}}$ and $v_{\mathrm{p}}=\sqrt{\mathrm{VXi}^{2}+\mathrm{VYi}^{2}+\mathrm{VZi}^{2}}$ are the $\mathrm{HI}$ mass and peculiar initial velocity of an HVC component, respectively. Population $\mathrm{A}-\mathrm{C}$ has two possible values of kinetic energy in accordance with the adopted solution for the distances. The total HI mass and kinetic energy of the system of HVCs are: (a) $M_{\mathrm{H}}^{\mathrm{a}}=3.42 \times 10^{8} M_{\odot}$ and $E_{\mathrm{K}}^{\mathrm{a}}=2.4 \times 10^{56} \mathrm{erg}$ considering the near- distance magnitudes for Complexes $\mathrm{A}$ and $\mathrm{C}$, and (b) $M_{\mathrm{H}}^{\mathrm{b}}=8.61 \times 10^{8} M_{\odot}$ and $E_{\mathrm{K}}^{\mathrm{b}}=5.3 \times 10^{56}$ erg considering the far-distance magnitudes for Complexes A and C. We assume that the HVCs have as much ionized as neutral hydrogen (Sembach et al. 2002; and Paper I). To obtain the mass of hydrogen nuclei (i.e. the sum of the hydrogen ions (free protons) and hydrogen atoms), we therefore multiply the above figures by two and, to derive the total gaseous mass, by a factor of 2.6, where we assume that the helium mass content is 30 percent of the hydrogen mass content.

We adopt Case a as the most probable scenario, since the Complexes $\mathrm{A}$ and $\mathrm{C}$ appear to be interacting with the Galactic edge. Brüns et al. (2000) showed that some HVCs of these complexes have a cometary appearance (see also Kalberla \& Haud 2006). A promising method to resolve the degeneracy in the distance is provided by the $\mathrm{H}_{\alpha}$ flux of the HVCs, which can act an indicator of distance. However, it is not simple to make inferences about the distances to the HVCs, since the origin of the HVC ionization is not well understood. The Galaxy's radiation field cannot account for the ionization degree of the MS (Putman et al. 2003). We suggest that HVCs are fossil HII regions and a large part of the HVC ionization is intrinsic, since they are likely to be relics of ionization fronts, strong stellar winds, and thermonuclear explosions of supernovae.

To compare the HI mass of the MS given in Table 1 with the mass obtained by Brüns et al. (2005), we estimate the root mean

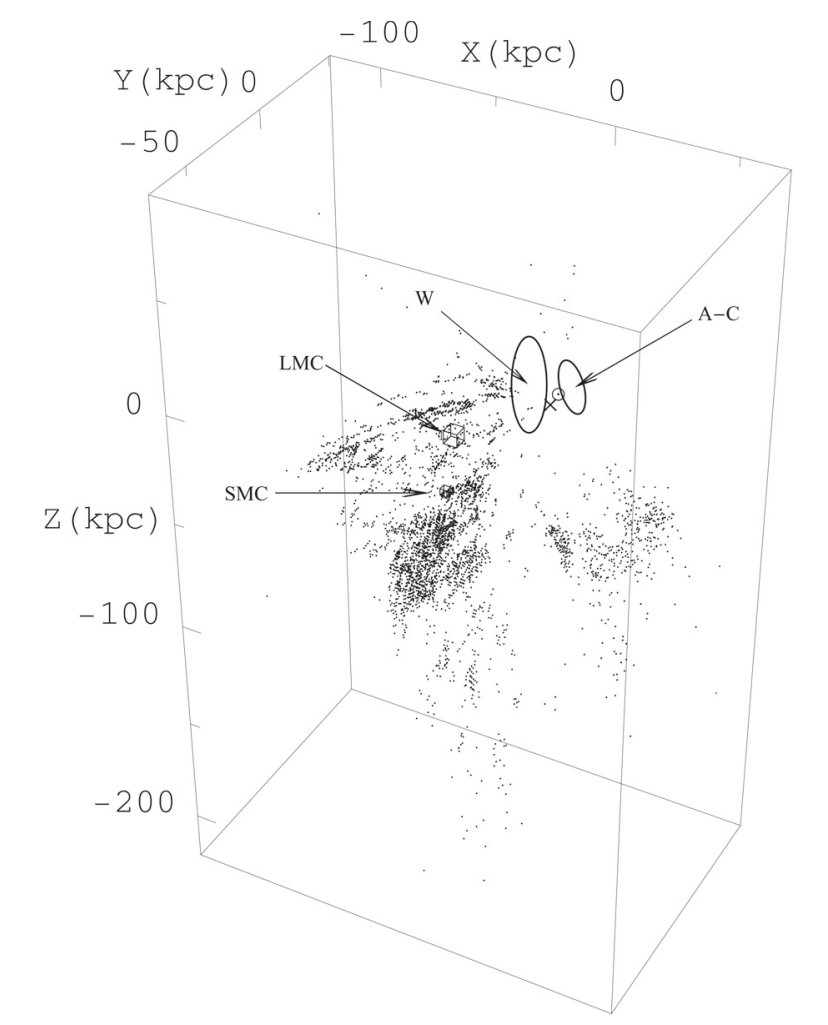

Fig. 20. Spatial distribution of the HVC populations. The dots represent the HVC-component positions of the main body of Population MS. The locations of Population W and Population A-C at its near distances are indicated schematically by ellipses. The positions of the LMC and SMC are represented.

square of the distances to the HVC components of the MS, $\sqrt{\overline{d^{2}}}$, at $81.5 \mathrm{kpc}$. According to our definition, the MS is connected to both the trailing Stream and the Leading Arm. From Table 3 of Brüns et al. (2005), we find that the HI mass of the MS is $1.54\left(\frac{81.5}{55}\right)^{2} \times 10^{8}=3.37 \times 10^{8} M_{\odot}$, which is in good agreement with our result. To provide a measure of the relative masses of the MCs main components, the HI masses taken from Table 3 of Brüns et al. (2005) are multiplied by 1.3 to take account of the helium content. The gas mass detached from the MCs is composed of the masses of the Magellanic bridge, $2.39 \times 10^{8} M_{\odot}$, and interface region, $1.94 \times 10^{8} M_{\odot}$, and the mass lost as HVCs $\left(2.6 M_{\mathrm{H}}^{a}=8.89 \times 10^{8} M_{\odot}\right.$, Case $\left.a\right)$. Hence, the gas masses outside the LMC and SMC amount to $\sim 1.32 \times 10^{9} M_{\odot}$, that is $12 \%$ of the MCs mass (the LMC mass $=0.87 \times 10^{10} M_{\odot}$, van der Marel et al. 2002 , and the SMC mass $=2 \times 10^{9} M_{\odot}$, Stanimirović et al. 2004). The total gas masses retained by the LMC and SMC are $5.73 \times 10^{8} M_{\odot}$ and $5.23 \times 10^{8} M_{\odot}$, that is $7 \%$ and $26 \%$ of their respective total masses. We neglect the $\mathrm{H}_{2}$ masses of the LMC 
and SMC, which are only $~ 10 \%$ of the HI masses (Fukui et al. 1999; Leroy et al. 2006)

\section{Physical and chemical links between the HVCs and the Magellanic Clouds}

An important characteristic of HVCs is their subsolar metallicities, which is indicative of a formation from gas poorly enriched with heavy elements. The chemical abundances provide a key constraint to associate HVCs with a parent object. The observed metallicities of the HVCs range from $Z=0.1$ to $0.3 Z_{\odot}$. Metallicity measurements of $0.25 \pm 0.07 Z_{\odot}$ (Lu et al. 1998) and $0.33 \pm 0.05 Z_{\odot}$ (Gibson et al. 2000) were found for the MS. The metallicities of the complex $\mathrm{C}$ vary from 0.1 to $0.3 Z_{\odot}$ (Richter et al. 2001a; Tripp et al. 2003). Collins et al. (2007) confirmed previous evidence for abundance variations across the cloud, which is independent of ionization effects, suggesting the possible mixing of primordial and enriched gas. The metallicity of the complex A was estimated to be $\sim 0.1 Z_{\odot}$, with a large uncertainty (Wakker 2001a).

The cloud-to-cloud variations of the observed metallicities, if significant, do not imply necessarily that the HVCs have diverse origins. This abundance scatter can be explained within the hypothesis of a common origin for the HVCs. First, it can reflect an inhomogeneous and/or anisotropic distribution of the abundances in the interstellar medium from which the gas of the HVCs originated. Second, the original abundances can be altered by the accretion of material swept by the HVCs along their trajectories. This is probably the case for the IVCs, whose $Z \sim 0.5-1 Z_{\odot}$ could be the result of material being accreted from the Galactic disk. Third, another cause of abundance evolution might be the selective loss of particles due to absorption onto dust grains and/or escape from the HVCs. There is evidence of dust in some HVCs, which is manifested by the presence of $\mathrm{H}_{2}$ in the MS (Richter et al. 2001b; Sembach et al. 2001) and by the dust emission in Complex C (Miville-Deschênes et al. 2005). On the other hand, we mention that the abundance ratios of depleted to undepleted elements in Complex $\mathrm{C}$ suggest that there is little dust present in Complex $\mathrm{C}$, if it is not absent completely (Wakker 2006).

We now investigate whether the overall abundance pattern of HVCs agrees with the chemical enrichment history of the MCs. Dopita et al. (1997) found that the base metallicity of the $\mathrm{LMC}, \sim 0.3 Z_{\odot}$, almost doubled $\sim 2 \mathrm{Gyr}$ ago. The present-day metallicity of the Magellanic bridge, $Z \approx 0.08 Z_{\odot}$, given by the abundances in the interstellar gas and young stars (Rolleston et al.1999; Lehner 2002) indicates the presence of an unenriched (primordial) gas component in the MCs, which could have been significant at the time of formation of the HVCs. The age-metallicity relation for the SMC shows that the mean metallicity of the SMC had an almost constant value of $\approx 0.1 Z_{\odot}$ from $10 \mathrm{Gyr}$ to $2 \mathrm{Gyr}$ ago, and during the last $2 \mathrm{Gyr}$ increased to $\approx 0.2 Z_{\odot}$ (Harris et al. 2004). This small increase in the metallicity of the SMC might be evidence that the SMC was fueled with fresh primordial gas that was accreted from the SMC periphery. This is evidence that the interstelar-medium of the MCs had a chemical composition similar to that of the HVCs, and that the HVCs can therefore be associated with the MCs. Until about 1 Gyr ago, when an important part of the gas of the MCs was lost as HVCs, the MC were primarily gaseous, with a high fraction of gas unprocessed by stars. The MCs, in a similar way to the majority of dwarf irregular galaxies, are gas-rich systems. Even today, 7 percent of the mass of the LMC and 26 percent of the mass of the SMC consists of gas, compared with only 2 percent of the mass of the Milky Way.

We present facts to support our idea that the HVCs were formed from gas dispersed from the MCs by the collective action of a large number of massive stars formed suddenly by the interaction between both Clouds. Several previuos studies found that the star formation in the LMC was almost constant for $10 \mathrm{Gyr}$, then increased by a factor of three during the past 2 Gyr (Gallagher et al. 1996; Holtzman et al. 1997; Westerlund et al. 1998). The star-formation history of the SMC is similar to that of the LMC. The sudden rise in the star-formation rate $2 \mathrm{Gyr}$ ago is reflected in a corresponding sudden rise in the metallicity (see above). There is evidence that this period of enhanced star formation is dominated by intermittent bursts. For instance, Elson et al. (1997) found that, in the inner disk of the LMC, a burst at about 1 Gyr possibly corresponded to the formation of the bar. Furthermore, Nidever et al. (2007) identified a specific site of the LMC, close to the edge of the LMC bar, and timescale of $\sim 1.3 \mathrm{Gyr}$ for the creation of the MS. We note that the timescale of these events, $\sim 1 \mathrm{Gyr}$, is coincident, within the errors, with the time estimated by ourselves for the crossing of the LMC and SMC centers (i.e. $0.8 \mathrm{Gyr}$ ). We suggest that the ISM turbulence, caused by the close encounter of the LMC and $\mathrm{SMC}$, increased the pressure on the preexisting giant molecular clouds of the inner galaxy, which triggered a chain of starformation events that probably culminated in the formation of the LMC bar. We hypothesize that the strong tidal forces and the shock wave associated with the passage of the SMC through the LMC, elongated and compressed the LMC inner disk of gas and stars, forming the bar of the LMC. This process triggered probably the formation of the starburst that occurred about 1 Gyr ago (Elson et al. 1997), in which $\sim 15 \%$ of the stellar mass of the bar was created (Smecker-Hane et al. 2002). The total mass of the bar $M_{\mathrm{b}}$ is about $10^{9} M_{\odot}$ (Zhao \& Evans 2000). We show that the number of massive stars formed by this burst can account for the large quantity of energy required to produce the HVCs. Using a Salpeter initial mass function (IMF), $\xi(m)=\xi_{0} m^{-2.35}$, and the condition $\int_{m_{0}}^{m_{1}} m \xi(m) \mathrm{d} m=15 \% M_{\mathrm{b}}$, we obtain the value of the constant $\xi_{0}$ and the total number of type II supernovae that exploded in the burst $n_{\mathrm{SN}}=\int_{m_{\mathrm{sn}}}^{m_{1}} \xi(m) \mathrm{d} m \sim 6.4 \times 10^{5}$, where $m_{0}=0.1 M_{\odot}, m_{1}=150 M_{\odot}$, and $m_{\mathrm{SN}}=20 M_{\odot}$ are the adopted values for the lower and upper limits of stellar mass, and the lower limit of type II supernova mass, respectively. The value of $n_{\mathrm{SN}}$ calculated above can explain the energy required to produce the HVCs (see below). Finally, we note that the most energetic regions of the burst, probably related to the origin of the HVCs, are not necessarily coincident with the densest stellar regions of the fossil starburst. If these regions produced predominantly massive stars, most of their stars have died exploding as type II supernovae a long time ago. Elmegreen (2004) (and references therein) indicated that some starbursts and clusters in other galaxies have initial mass functions (IMFs) biased toward massive stars. A natural theoretical interpretation of these "top-heavy" IMFs is that the increase in the molecular-cloud turbulence due to the star-formation rate, and hence the energy released by supernovae, increases the Jeans mass.

The scenario described in the previous paragraph involves the inner galaxies. By the time of the LMC-SMC collision, probably each galaxy had two different regions: the inner galaxy populated by stars, clouds of enriched gas, and GMCs, and the outer galaxy composed essentially of primordial gas (or 
less-enriched gas). This interpretation is supported by the observation that the metallicity across the LMC decreases outward radially (Kontizas et al. 1993; Bica et al. 1998; Cioni \& Habing 2003; Alves 2004). We describe a second scenario in which the collision of the extended and massive gaseous envelopes of the LMC and SMC likely played an important role in the origin of the HVCs. The outer edges of the MCs had probably similarities with the primitive environments in which the first stars (population III) were formed. It is widely accepted in theoretical studies that the first stars were predominantly massive (Bromm $\&$ Larson 2004). The essentially pure hydrogen clouds of the outer regions of both Clouds might have remained in hydrostatic quasi-equilibrium, due to inefficient cooling, until the Clouds collided. The compression of the gaseous envelopes of the LMC and SMC may have induced cloud-cloud collisions in which efficient three-body processes tranformed $\mathrm{H}$ into $\mathrm{H}_{2}$ (Palla et al. 1983), the main coolant of these clouds, permitting the coalescence of the colliding clouds. In this way, the clouds may have grown in mass and eventually reached the Jeans mass. Therefore, bursts of massive-star formation triggered by collisions between sufficiently massive gaseous envelopes of galaxies, appear to be quite possible. The massive stars evolved in a few Myr exploding as type II supernovae and hence the fossil burst of massive stars is not identifiable today by means of the observation of normal stars.

In this context, the number of massive stars, and hence of supernovae, formed in the starburst that produced the HVCs, can be estimated by means of $n_{\mathrm{SN}}=\frac{E_{\mathrm{k}}}{E_{\mathrm{SN}}}$, where $n_{\mathrm{SN}}$ is the number of type II supernovae, $E_{\mathrm{k}}$ is the total kinetic energy of the HVCs, and $E_{\mathrm{SN}}$ is the kinetic energy of the expanding ejecta of a type II supernova. Adopting $E_{\mathrm{k}}=2.6 E_{\mathrm{k}}^{a}=6.24 \times 10^{56} \mathrm{erg}$ and $E_{\mathrm{SN}}=1.7 \times 10^{51} \mathrm{erg}$ (Arnett 1996), $n_{\mathrm{SN}} \sim 3.6 \times 10^{5}$. A large part of the MC's primordial gas was polluted by the products of these supernovae and expelled as HVCs. Hence, the absolute metal abundances of the HVCs is given approximately by $Z=\frac{n_{\mathrm{SN}} y\left(M_{\odot}\right)}{M_{\mathrm{H}}\left(M_{\odot}\right)} W$, where $y$ is the yield of Fe (or $\left.{ }^{56} \mathrm{Ni}\right)$ per supernova, and $M_{\mathrm{H}}$ is the total mass of hydrogen nuclei of the HVCs, and $W=1 / 56$, the ratio of the relative atomic weights of $\mathrm{H}$ and Fe. To determine the abundance relative to solar abundance metallicity, the previous formula for $Z$ was divided by $Z_{\odot}=3.24 \times 10^{-5}$ (Wakker 2001a). Assuming $M_{\mathrm{H}}=2 M_{\mathrm{H}}^{a}=6.8 \times 10^{8} M_{\odot}$ and $y=0.075 M_{\odot}$ (Arnett 1996), we obtained $Z=0.02 Z_{\odot}$. We interpret this number for $Z$ as a minimum mean value of the HVC metal abundances because of the assumption that supernova remnants were mixed with pristine primordial gas. Another fact supporting our arguments is the enhancement of the $\alpha$-elements/Fe ratio observed in the complexes $\mathrm{A}$ and $\mathrm{C}$, a nucleosynthesis signature of type II supernovae (Wakker 2001b; Collins et al. 2007; Gibson 2007).

The two extreme scenarios presented above are not mutually exclusive, and may have occurred in combination. The abundance enrichment $\Delta Z$ due to the synthesized products of the massive stars generated by the LMC-SMC collision was $\sim 0.02 Z_{\odot}$, that is the abundance increment added to the then-existing gas. Hence, the observed abundances of the HVCs, $\sim 0.1-0.3 Z_{\odot}$, can be explained by partial mixture of the inner galaxy clouds with the outer galaxy ones. We propose that the LMC-SMC collision mixed parts of the gas envelopes with the inner galaxy clouds, disrupted less dense clouds and collapsed the denser ones into massive stars, which in turn completed the process of contamination, mixture and disruption of a large part of the gaseous material of the MCs

\section{Summary and conclusions}

According to Paper I, the majority of HVCs were ejected from the MCs probably as a consequence of a strong interaction between the Clouds that induced a burst of formation of massive stars and subsequent stellar winds and supernova explosions. Following this idea, we developed a kinematical model and two dynamical models that allowed us to obtain the spatial distribution of the HVCs from the observational data of the HVCs, that is the sky positions and radial velocities of their HI components. From the kinematical model, we obtained a simple analytical formula that provided the distances to the HVCs with an internal precision of a few kpc. This formula was

$$
\begin{aligned}
d= & 550(\rho+133+220 \sin l \cos b \\
& \left.+282 \cos \left(l-78^{\circ}\right) \cos \left(b-2^{\circ}\right)\right) \\
& +50.1 \times 10^{3}\left(\cos b \cos \left(-33^{\circ} .9\right) \cos \left(l-280^{\circ} .5\right)\right. \\
& \left.+\sin b \sin \left(-33^{\circ} .9\right)\right)
\end{aligned}
$$

where the distance $d$, the observed radial velocity $\rho$, and the galactic position $(l, b)$ of the HVC were expressed in $\mathrm{pc}, \mathrm{km} \mathrm{s}^{-1}$ and degrees, respectively.

The first step in the study of the dynamical evolution of the MC-HVC system is to determine the gravitational potential of the Milky Way. With this objective, we developed two dynamical models: Model 1, in which the Galactic potential was determined by a spherical halo of dark matter; and Model 2, in which in addition a massive thick disk with dark matter contributed to the potential. With the constraint that the models reproduced as closely as possible, observational data of the MCs and HVCs, such as that the MCs collided and that a component of Complex A lies at $\approx 10 \mathrm{kpc}$ from the Sun, we were able to place constraints on the free parameters of the Models. The Milky Way mass within, a radius of $50 \mathrm{kpc}$ from the Galactic center, and the time at which the MCs collided, were found to be $3.9 \times 10^{11} M_{\odot}$ and $840 \mathrm{Myr}$ ago, respectively, according to the Model 1 results, and $4.6 \times 10^{11} M_{\odot}$ and $720 \mathrm{Myr}$ ago, respectively, according to those of Model 2. The time at which the LMC and SMC collided, provided the origin time of the HVCs.

The method for determining distances to the HVCs in our dynamical models was as follows. With the initial conditions common to all HVCs and boundary conditions imposed by an assumed distance to the HVC under study, the motion equations were solved for the remaining unknowns of the HVC (i.e. the peculiar initial velocity and the present velocity). Thus, we constructed a theoretical relationship between the distance and the radial velocity along the line of sight to each HVC component. Then, we asked for the value of the HVC distance that the corresponding theoretical relationship assigned to the value of the argument, that is the observed radial velocity of the HVC.

In the light of our dynamical models, the HVCs could be grouped into three populations, that we called $\mathrm{MS}, \mathrm{W}$ and $\mathrm{A}-\mathrm{C}$. All HVCs of the first and second Galactic quadrants belong to Population A-C, apart from the southern HVCs that belong to the "classic" Magellanic stream. Population A-C is the only one for which the distance measurement was ambiguous, since its distance had two possible solutions, a near distance of about $6 \mathrm{kpc}$, and a far distance ranging from 5 to $250 \mathrm{kpc}$. For the fardistance solution, Population $\mathrm{A}-\mathrm{C}$ formed a huge structure in the shape of doughnut. Although from the dynamical perspective we cannot discard the far-distance solutions for Population A-C, evidence that the HVCs of this population are likely interacting with the outer Galactic disk favors its near-distance solutions. Further evidence is that an HVC 
component of the Cohen stream, likely a southern extension of Population A-C, has a distance between 5 to $11.7 \mathrm{kpc}$. Hence, Fig. 20 could illustrate the probable spatial distribution of the HVCs.

Population W is a group of positive-velocity HVCs located in the Galactic periphery of the third and fourth quadrants at a mean distance of about $15 \mathrm{kpc}$. The movement of the HVCs of Population $\mathrm{W}$ throughout the gas layer of the outer Galactic disk gave rise to frictional forces that strongly braked these HVCs. Population MS was distributed about the LMC and SMC in a structure, flattened towards the orbital plane of the MCs (i.e. almost the $\mathrm{X}-\mathrm{Z}$ plane), whose main body was enclosed by a disk of a diameter of $\approx 200 \mathrm{kpc}$ and a thickness of $\approx 60 \mathrm{kpc}$. The mean density of this structure was about 10 atoms $\mathrm{m}^{-3}$.

The kinematic distances provided by the analytical formula (Eq. (20)), as compared with the dynamical distances, gave good approximations to the distances of Population MS and the far distances of Population A-C. However, Eq. (20) could not be applied to obtain the distances of Population $\mathrm{W}$ and the near distances of Population A-C. In these two cases, the trajectories of the HVCs were perturbed by the interaction with the Galactic gas layer and the dynamical models should be used.

In accordance with optical studies, we suggested that the collision between the LMC and SMC triggered a major burst of star formation that gave origin to a stellar component of the LMC bar. The star burst formed a large number of massive stars, whose total energy deposited into the LMC interstellar medium through expanding HII regions, stellar winds, and supernova explosions, produced the kinetic energy of HVC system, $\approx 6 \times 10^{56} \mathrm{erg}$. Finally, we showed that the low metallicities observed in the HVCs are compatible with the hypothesis of a Magellanic Cloud origin for the HVCs, reflecting the chemical conditions of the LMC and SMC interstellar gas from which the HVCs would have been formed.

Acknowledgements. I want to dedicate this work to the memory of Dr. Virpi S. Niemela, who helped me in difficult times of my career. The helpful comments of an anonymous referee led to substantial improvements in the paper. I would also like to thank the A\&A editor, C.M. Walmsley, for his help. Part of this work was supported by the Consejo Nacional de Investigaciones Cientificas y Técnicas (CONICET) project number PIP-5697.

\section{Appendix A: Procedure for adjusting the free parameters of the Galactic potential}

The HVCs can be considered as test particles for determining the free parameters of the Galactic potential, that is the scale radius $r_{\mathrm{h}}$ of the halo as well as the scale height $b_{\mathrm{d}}$ of a dark matter component associated with the disk in the case of Model 2. This is strictly true only if the non-gravitational forces affecting the trajectories of HVCs are negligible or well-known. We obtained the common initial conditions of the HVCs, $t_{\mathrm{e}}, \boldsymbol{r}_{\mathrm{L}}\left(t_{\mathrm{e}}\right)$ and $\dot{r}_{\mathrm{L}}\left(t_{\mathrm{e}}\right)$, as functions of $r_{\mathrm{h}}$ from Condition 1. These functions are represented in Figs. 3 and 5 for Model 1. In Model 2, these functions depend also on $b_{\mathrm{d}}$, and thus we represent them for the adopted value $b_{\mathrm{d}}=3520$ pc (Figs. 6 and 8). Hence, the value of $r_{\mathrm{h}}$ (and that of $b_{\mathrm{d}}$ in Model 2) determines the predicted distance of an HVC and should be such that Condition 2 is satisfied. In Sects. 3.1.1 and 3.2.1, we used the results of the $r_{\mathrm{h}}$ fitting without explaining its procedure. To do this, we need to apply a variant of the method described in Sect. 3.3 as follows.

Since we know the distance to the upper end of Complex A $\left(l=160^{\circ} .4, b=43^{\circ} .3\right)$, estimated by direct methods at $\approx 4-11 \mathrm{kpc}$, we use this fact to determine the value of $r_{\mathrm{h}}$. Given $d$ and $(l, b)$, we construct a relationship between $\rho$ and $r_{\mathrm{h}}$. We adopt
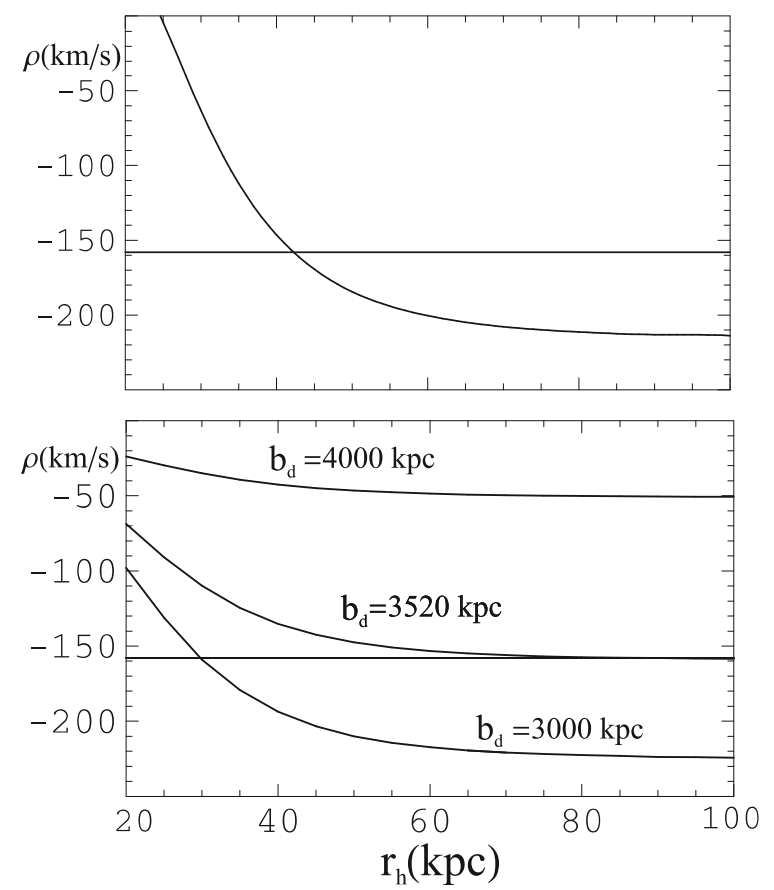

Fig. A.1. Upper panel: $\rho-r_{\mathrm{h}}$ relationship of Model 1 corresponding to the Complex A distance $(d=11 \mathrm{kpc})$ at the position $(l=160.4, b=$ 43.3). The abscissa value of the intersection point of the straight line $\rho=-158 \mathrm{~km} \mathrm{~s}^{-1}$ (the radial velocity of Complex A at that position) and the $\rho-r_{\mathrm{h}}$ curve gives us $r_{\mathrm{h}}=42 \mathrm{kpc}$. Lower panel: same as the upper one, but for Model 2. Given the total mass of the Galactic disk $M_{\mathrm{d}}$ and its surface density at the solar position, $\Sigma$, the $\rho-r_{\mathrm{h}}$ relationship depends on a scale height $b_{\mathrm{d}}$ representing the thickness of the Galactic disk of dark matter. We plot this relationship for three values of $b_{\mathrm{d}}$ (indicated on the respective curves). The curves were calculated with $M_{\mathrm{d}}=3 \times$ $10^{11} M_{\odot}$ and $\Sigma_{1.1}=74 M_{\odot} \mathrm{pc}^{-2}$, therefore $b_{\mathrm{d}}=3000,3520$ and $4000 \mathrm{pc}$ correspond the scale radius of the disk $a_{\mathrm{d}}=9548,7614$ and $5661 \mathrm{pc}$ respectively.

the upper limit of distance as the distance to this HVC component, because this is close to the bifurcation-point distance and hence it is not necessary to include the drag forces in the calculations. Unlike the construction of the $\rho-d$ relationships in which we kept $r_{\mathrm{h}}=$ const. and varied the distances at regular intervals (see Sect. 3.3), we keep $d=11 \mathrm{kpc}$, vary $r_{\mathrm{h}}$ at regular intervals, and assign, in the case of Model 2, an arbitrary value to $b_{\mathrm{d}}$. This $\rho-r_{\mathrm{h}}$ relationship assigns the value of $r_{\mathrm{h}}$ to the observed radial velocity of Complex $A$ at that position, $-158 \mathrm{~km} \mathrm{~s}^{-1}$ (see Fig. A.1). In the case of Model 2 , we vary $b_{\mathrm{d}}$ to obtain the solutions for $r_{\mathrm{h}}$ as a function of $b_{\mathrm{d}}$.

The upper panel of Fig. A.1 gives the $\rho-r_{\mathrm{h}}$ relationship of Model 1, which determines $r_{\mathrm{h}}=42 \mathrm{kpc}$. We calculate the $\rho-r_{\mathrm{h}}$ relationship of Model 2 for different values of $b_{\mathrm{d}}$ (see the lower panel of Fig. A.1). The curves for which $b_{\mathrm{d}}>3520 \mathrm{pc}$ do not intersect the straight line, and hence are incompatible with Condition 2. We adopt the curve that has the highest value of $b_{\mathrm{d}}$ compatible with Condition 2, i.e. $b_{\mathrm{d}}=3520 \mathrm{pc}$, which is the closest value to that of $4000 \mathrm{pc}$ estimated by Kalberla et al. (2007), and implies a halo as massive as the disk. The scale radius of the Galactic disk corresponding to $b_{\mathrm{d}}=3520 \mathrm{pc}$ is $a_{\mathrm{d}}=7614 \mathrm{pc}$. In this case $r_{\mathrm{h}}$ is ill-determined and has values of the order of $100 \mathrm{kpc}$ or greater. We adopt $r_{\mathrm{h}}=100 \mathrm{kpc}$ for Model 2. The initial conditions represented in Figs. 6 and 8 are almost independent of the value of $r_{\mathrm{h}}$, for $r_{\mathrm{h}} \geq 100 \mathrm{kpc}$. Therefore, the chosen value for $r_{\mathrm{h}}$ within this range is irrelevant. This is because the mass and extent of the halo beyond the orbits 
of the MCs do not have net gravitational influence on the interior region so long as the halo is homogeneous and spherical or ellipsoidal.

If we use in Model 2 the mass distribution of the Galactic disk assigned to the visible matter, the corresponding $\rho-r_{\mathrm{h}}$ relationships do not intersect the line $\rho=-158 \mathrm{kms}^{-1}$. Therefore, Model 2 without any dark matter within the disk, produces results that are inconsistent with observational constraints.

Our method will benefit from further direct determinations of accurate distance constraints for HVCs, and high-quality determinations of the MCs proper motions with a precision 5-10 times better than the precision achieved today. In this regard, some progress has been made recently in determining distance approximations for Complex C (Thom et al. 2007; Wakker et al. 2007) and for Cohen's Stream, Complex GCP and Cloud g1 (Wakker et al. 2008). The inclusion of further boundary conditions to be satisfied by the models will permit a better fitting of the free parameters, and add new parameters to be fitted. These new free parameters could be for instance those characterizing the distribution of the Galactic coronal gas for a phenomenological approach to the treatment of drag forces acting on the HVCs.

\section{References}

Alves, D. R. 2004 [arxiv: astro-ph/0408336]

Arnett, D. 1996, Supernovae and nucleosynthesis (Princeton Univ. Press)

Bekki, k., \& Chiba, M. 2007, MNRAS, 381, L16

Benjamin, R. A., \& Danly, L. 1997, ApJ, 481, 764

Besla, G., Kallivayalil, N., Hernquist, L., et al. 2007, ApJ, 668, 949

Bica, E., Geisler, D., Dottori, H., et al. 1998, AJ, 116, 723

Binney, J., \& Merrified, M. 1998, Galactic astronomy (Princeton Univ. Press) Binney, J., \& Tremaine, S. 1987, Galactic dynamics (Princeton Univ. Press) Blitz, L., Spergel, D. N., Teuben, P. J., Hartman, D., \& Burton, W. B. 1999, ApJ, 514,818

Bregman, J. N. 1980, ApJ, 236, 577

Bromm, V., \& Larson, R. B. 2004, ARA\&A, 42, 79

Brüns, C., Kerp, J., Kalberla, P. M. W., \& Mebold, U. 2000, A\&A, 357, 120

Brüns, C., Kerp, J., Staveley-Smith, L., et al. 2005, A\&A, 432, 45

Cioni, M.-R. L., \& Habing, H. J. 2003, A\&A, 402, 133

Collins, J. A., Shull, J. M., \& Giroux, M. L. 2007, ApJ, 657, 271

Comerón, F., \& Torra, J. 1992, A\&A, 261, 94

Connors, T. W., Kawata, D., \& Gibson, B. K. 2006, MNRAS, 371, 108

de Boer, W., Sander, C., Zhukov, V., Cladyshev, A. V., \& Kazakov, D. I. 2005,

A\&A, 444, 51

Dopita, M. A., Vassiliadis, E., Wood, P. R., et al. 1997, ApJ, 474, 188

Elmegreen, B. G. 2004, MNRAS, 354, 367

Elson, R. A. W., Gilmore, G. F., \& Santiago, B. X. 1997, MNRAS, 289, 157

Fukui, Y., Mizuno, N., Yamaguchi, R., et al. 1999, PASJ, 51, 745

Gallagher, J. S., Mould, J. R., de Feijter, E., et al. 1996, ApJ, 466, 732

Gardiner, L. T., \& Noguchi, M. 1996, MNRAS, 278, 191

Gibson, B. K. 2007, Proc. IAU Symp., 241, ed. A. Vazdekis, \& R. F. Peletier (Cambridge Univ. Press), 161

Gibson, B. K., Giroux, M. L., Penton, S. V., et al. 2000, AJ, 120, 1830

Giovanelli, R. 1981, AJ, 86, 1468

Harris, J., \& Zaritsky, D. 2004, AJ, 127, 1531

Heiles, C. 1984, ApJS, 55, 585

Hernquist, L. 1993, ApJS, 86, 389

Holtzman, J. A., Mould, J. R., Gallagher III, J. S., et al. 1997, AJ, 113, 656

Hopp, U., Schulte-Ladbeck, R. E., \& Kerp, J. 2007, MNRAS, 374, 1164

Hulsbosch, A. N. M., \& Oort, J. H. 1973, A\&A, 22, L153

Kalberla, P. M. W. 2003, ApJ, 588, 805

Kalberla, P. M. W., \& Haud, U. 2006, A\&A, 455, 481

Kalberla, P. M. W., Dedes, L., Kerp, J., \& Haud, U. 2007, A\&A, 469, 511

Kallivayalil, N., van der Marel, R. P., \& Alcock, C. 2006, ApJ, 652, 1213

Kochanek, C. S. 1996, ApJ, 457, 228

Kontizas, M., Kontizas, E., \& Michalitsianos, A. G. 1993, A\&A, 269, 107

Kroupa, P., \& Bastian, U. 1997, New Astron., 2, 77

Kuntz, K. D., \& Danly, L. 1996, ApJ, 457, 703

Lehner, N. 2002, ApJ, 578, 126

Leroy, A., Bolatto, A., Stanimirović, S., et al. 2007 ApJ, 658, 1027
Lin, D. N. C., Jones, B. F., \& Klemola, A. R. 1995, ApJ, 439, 652

Lu, L., Sargent, W. L. W., Savage, B. D., et al. 1998, AJ, 115, 162

Lynden-Bell, D., Cannon, R. D., \& Godwin, P. J. 1983, MNRAS, 204, 87P

Maller, A. H., \& Bullock, J. S. 2004, MNRAS, 355, 694

Mastropietro, C., Moore, B., Mayer, L., Wadsley, J., \& Stadel, J. 2005, MNRAS, 363,509

Meurer, G. R., Bicknell, G. V., \& Gingold, R. A. 1985, PASAu, 6, 195

Mirabel, I. F. 1981, ApJ, 250, 528

Mirabel, I. F. 1982, ApJ, 256, 112

Miville-Deschênes, M. A., Boulanger, F., Reach, W. T., \& Noriega-Crespo, A. 2005, ApJ, 631, L57

Miyamoto, M., \& Nagai, R. 1975, PASJ, 27, 533

Momany, Y., Zaggia, S., Gilmore, G., et al. 2006, A\&A, 451, 515

Moore, B., \& Davis, M. 1994, MNRAS, 270, 209

Murai, T., \& Fujimoto, M. 1980, PASJ, 32, 581

Nidever, D. L., Majewski, S. R., \& Burton, W. B. 2007 [arXiv: 0706. 1578v1]

Olano, C. A. 2004, A\&A, 423, 895 (Paper I)

Oort, J. H. 1966, BAN, 18, 421

Palla, F., Salpeter, E. E., \& Stahler, S. W. 1983, ApJ, 271, 632

Peek, J. E. G., Putman, M. E., McKee, C. F., Heiles, C., \& Stanimirović, C. 2006, ApJ, 656, 907

Peek, J. E. G., Putman, M. E., \& Sommer-Larsen, J. 2007 [arXiv:0705.0357v2]

Pisano, D. J., Barnes, D. G., Gibson, B. K., et al. 2004, ApJ, 610, L17

Putman, M. E., Bland-Hawthorn, J., Veilleux, S., et al. 2003, ApJ, 597, 948

Richter, P. 2006, Reviews in Modern Astronomy, ed. R. Siegfried (Wiley-VCH Verlag), 19, 31

Richter, P., Sembach, K. R., Wakker, B. P., et al. 2001a, ApJ, 559, 318

Richter, P., Sembach, K. R., Wakker, B. P., \& Savage, B. D. 2001b, ApJ, 562, L181

Rolleston, W. R. J., Dufton, P. L., McErlean, N. D., \& Venn, K. A. 1999, A\&A, 348,728

Santillán, A., Franco, J., Martos, M., \& Kim, J. 1999, ApJ, 515, 657

Sembach, K. R., Savage, B. D., \& Massa, D. 1991, ApJ, 372, 81

Sembach, K. R., Howk, J. C., Savage, B. D., \& Shull, J. M. 2001, AJ, 121, 992

Sembach, K. R., Gibson, B. K., Fenner, Y., \& Putman, M. E. 2002, ApJ, 572, 178

Shapiro, P. R., \& Field, G. B. 1976, ApJ, 205, 762

Siegel, M. H., Majewski, S. R., Gallart, C., et al. 2005, ApJ, 623, 181

Simon, J. D., \& Blitz, L. 2002, ApJ, 574, 726

Smecker-Hane, T. A., Cole, A. A., Gallagher, J. S., \& Stetson, P. B. 2002, ApJ, 566,239

Sommer-Larsen, J. 2006, ApJ, 644, L1

Stanimirović, S., Staveley-Smith, L., \& Jones, P. A. 2004, ApJ, 604, 176

Tamanaha, C. M. 1995, ApJ, 450, 638

Tamanaha, C. M. 1997, ApJS, 109, 139

Tenorio-Tagle, G. 1980, A\&A, 88, 61

Tenorio-Tagle, G. 1981, A\&A, 94, 338

Thom, C., Putman, M. E., Gibson, B. K., et al. 2006, ApJ, 638, L97

Thom, C., Peek, J. E. G., Putman, M. E., et al. 2007 [arXiv:0712.0612v1]

Tripp, T. M., Wakker, B. P., Jenkins, E. B., et al. 2003, AJ, 125, 3122

van der Marel, R. P., Alves, D. R., Hardy, E., \& Suntzeff, N. B. 2002, AJ, 124, 2639

van Woerden, H., Schwarz, U. J., Peletier, R. F., Wakker, B. P., \& Kalberla, P. M. W. 1999, Nature, 400, 138

van Woerden, H., Wakker, B. P., Schwarz, U. J., \& de Boer, K. S. 2004, High Velocity Clouds (Kluwer Academic Publishers), ASSL, 312

Wakker, B. P. 2001a, ApJS, 136, 463

Wakker, B. P. 2001b [arXiv: astro-ph/0109210v1]

Wakker, B. P. 2004, High Velocity Clouds, ASSL, 312, 25

Wakker, B. P. 2006, ApJS, 163, 282

Wakker, B. P., \& van Woerden, H. 1991, A\&A, 250, 509

Wakker, B. P., \& van Woerden, H. 1997, ARA\&A, 35, 217

Wakker, B. P., Howk, J. C., van Woerden, H., et al. 1998, ASP Conf. Ser., 143, 280

Wakker, B. P., York, D. G., Howk, J. C., et al. 2007, ApJ, 670, L113

Wakker, B. P., York, D. G., Wilhelm, R., et al. 2008, ApJ, 672, 298

Wannier, P., Wrixon, G. T., \& Wilson, R. W. 1972, A\&A, 18, 224

Wesselius, P. R., \& Fejes, I. 1973, A\&A, 24, 15

Westerlund, B. E., Lundgren, K., Pettersson, B., \& Koziej, E. 1998, A\&A, 339, 385

Xue, X.-X., Rix, H.-W, Zhao, G., et al. 2008 [arXiv:0801.1232v2]

Yoshizawa, A. M., \& Noguchi, N. 2003, MNRAS, 339, 1135

Zhao, H. S., \& Evans, N. W. 2000, ApJ, 545, L35

Zwaan, M. A. 2001, MNRAS, 325, 1142 